\title{
SENSOR-BASED LONG BASELINE NAVIGATION: OBSERVABILITY ANALYSIS AND FILTER DESIGN
}

\author{
Pedro Batista, Carlos Silvestre, and Paulo Oliveira
}

\begin{abstract}
This paper presents a novel long baseline (LBL) position and velocity navigation filter for underwater vehicles based directly on the sensor measurements. The solution departs from previous approaches as the range measurements are explicitly embedded in the filter design, therefore avoiding inversion algorithms and allowing also the consideration of the cases of reduced numbers of readings, in particular when there are only two or three distance measurements. The nonlinear system dynamics are considered to their full extent and no linearizations are carried out whatsoever. The filter error dynamics are globally exponentially stable (GES) and it is shown, in a realistic simulation environment, that the filter achieves similar performance to the extended Kalman filter (EKF) and outperforms linear position and velocity filters based on algebraic estimates of the position obtained from the range measurements.
\end{abstract}

Key Words: Navigation, marine robotics, long baseline, nonlinear observability analysis.

\section{INTRODUCTION}

Accurate navigation systems are essential for the successful operation of autonomous vehicles. Although there exist alternatives such as terrain-based navigation, most navigation systems contain an inertial navigation system (INS) that provides the state of the vehicle by integrating, in openloop, the information provided by inertial sensors, e.g., accelerometers and rate gyros. Although INS provides very good short term results, its performance necessarily degrades over time as it corresponds to an open-loop integration system. In order to improve the navigation performance, aiding devices have been considered in the design of integrated navigation solutions. This paper addresses the problem of vehicle navigation using ranges to a set of landmarks arranged in a long baseline (LBL) configuration.

Among the myriad of aiding devices, the Global Positioning System (GPS) is a very popular choice [1-5]. For underwater vehicles, GPS is not a solution due to the strong attenuation that the electromagnetic field suffers in water. Therefore, other solutions have been sought in the past, includ-

Manuscript received November 27, 2012; revised April 3, 2013; accepted June 15, 2013 .

The authors are with the Institute for Systems and Robotics, Laboratory of Robotics and Systems in Engineering and Science, Portugal. Pedro Batista is also with Instituto Superior Técnico, Universidade de Lisboa, Portugal. Carlos Silvestre is also with the Department of Electrical and Computer Engineering, Faculty of Science and Technology of the University of Macau. Paulo Oliveira is also with the Department of Mechanical Engineering, Instituto Superior Técnico, Universidade de Lisboa, Portugal.

Pedro Batista is the corresponding author (e-mail: pbatista@isr.ist.utl.pt).

This work was partially supported by the FCT [PEst-OE/EEI/LA0009/2013] and by the EU Project TRIDENT (Contract No. 248497)

The authors are indebted to the anonymous reviewers for their contributions and, in particular, for the suggestion that resulted in Remark 7. ing acoustic positioning systems like LBL and short baseline (SBL) [6-9], and references therein. In [10] the author proposes a GPS-like system consisting of buoys equipped with differential GPS. A related solution, denominated as the GPS Intelligent Buoy (GIB) system, is now commercially available, see [11]. Further work on the GIB underwater positioning system can be found in [12]. Position and linear velocity global asymptotic stability (GAS) filters based on an ultra-short baseline (USBL) positioning system were presented by the authors in [13], while the extended Kalman filter (EKF) is the workhorse of the solution presented in [14]. Single range navigation solutions can be found in [15-24]. For interesting discussions and more detailed surveys on underwater vehicle navigation and sensing devices see $[25,26]$.

This paper presents a position and linear velocity navigation filter based on range measurements to fixed landmarks arranged in an LBL configuration. Traditional solutions resort either to the EKF or to solutions based on position algebraic estimates obtained from the range measurements. The solution presented in the paper departs from previous approaches as the range measurements are explicitly embedded in the filter design, therefore avoiding inversion algorithms. Moreover, the nonlinear system dynamics are considered to their full extent and no linearizations are carried out whatsoever, which allows us to show that the filter error dynamics are globally exponentially stable (GES). Central to the proposed filtering framework is the derivation of a nonlinear system that can be regarded as linear time-varying (LTV) and that captures the dynamics of the original nonlinear system. As the range measurements are directly embedded in the system dynamics, the present solution also allows us to consider two and three range measurements, which is not possible in 
solutions that resort to the computation of an algebraic estimate of the position from the range measurements. Applications of the proposed solution are many and, in a realistic simulation environment, it is shown that the filter achieves similar performance to the extended Kalman filter (EKF) and outperforms linear position and velocity filters based on position algebraic estimates obtained directly from the range measurements. Preliminary work on this subject by the authors can be found in [27], where the LBL sensor-based solution was first proposed. This paper presents complete and detailed proofs regarding this subject and also extends the results to the case of two and three measurements. In addition, the performance of the proposed solution is carefully evaluated and compared with traditional solutions. The specific case of aided navigation based on single range measurements has been addressed by the authors in [28-30]. A USBL sensor-based solution to the problem of underwater navigation, which resorts to similar tools as this paper, is proposed in [31].

The paper is organized as follows. The problem statement and the nominal system dynamics are introduced in Section II, while the filter design is detailed in Section III. Simulation results are presented in Section IV and the specific cases of two and three range measurements are considered in Section V. Finally, Section VI summarizes the main conclusions and results of the paper.

Notation. Throughout the paper the symbol $\mathbf{0}_{n \times m}$ denotes an $n \times m$ matrix of zeros, $\mathbf{I}_{n}$ an identity matrix with dimension $n \times n$, and $\operatorname{diag}\left(\mathbf{A}_{1}, \ldots, \mathbf{A}_{\mathrm{n}}\right)$ a block diagonal matrix. When the dimensions are omitted the matrices are assumed to be of appropriate dimensions. For $\mathbf{x} \in \mathbb{R}^{3}$ and $\mathbf{y} \in \mathbb{R}^{3}, \mathbf{x} \times \mathbf{y}$ and $\mathbf{x} \cdot \mathbf{y}$ represent the cross and inner products, respectively. Finally, the Dirac delta function is denoted by $\delta(t)$.

\section{PROBLEM STATEMENT}

Consider an underwater vehicle moving in a scenario where there is a set of fixed landmarks arranged in an LBL configuration, where acoustic beacons or transponders are installed, and suppose that the vehicle measures the range to each of the landmarks, as depicted in Fig. 1. Further assume that the vehicle is equipped with an inertial measurement unit (IMU), consisting of two triads of orthogonally mounted accelerometers and rate gyros, and an attitude and heading reference system (AHRS). The problem considered in the paper is the design of a sensor-based filter with globally exponentially stable error dynamics to estimate the position and linear velocity of the vehicle.

\subsection{System dynamics}

In order to detail the problem framework, let $\{I\}$ denote a local inertial reference coordinate frame and $\{B\}$ a coordinate frame attached to the vehicle, usually denominated as the body-fixed reference frame. The linear motion of the vehicle is given by

$$
\dot{\mathbf{p}}(t)=\mathbf{R}(t) \mathbf{v}(t),
$$

where $\mathbf{p}(t) \in \mathbb{R}^{3}$ denotes the inertial position of the vehicle, $\mathbf{v}(t) \in \mathbb{R}^{3}$ is the velocity of the vehicle relative to $\{I\}$, expressed in body-fixed coordinates, and $\mathbf{R}(t) \in S O(3)$ is the rotation matrix from $\{B\}$ to $\{I\}$, which satisfies $\dot{\mathbf{R}}(t)=\mathbf{R}(t) \mathbf{S}(\boldsymbol{\omega}(t))$, where $\omega(t) \in \mathbb{R}^{3}$ is the angular velocity of $\{B\}$, expressed in body-fixed coordinates, and $\mathbf{S}(\omega) x$ is the skew-symmetric matrix such that $\mathbf{S}(\omega)$ is the cross product $\omega \times x$.

The AHRS provides the rotation matrix $\mathbf{R}(t)$, while the IMU measures both the angular velocity $\omega(t)$ and the linear acceleration $\mathbf{a}(t)$, which satisfies

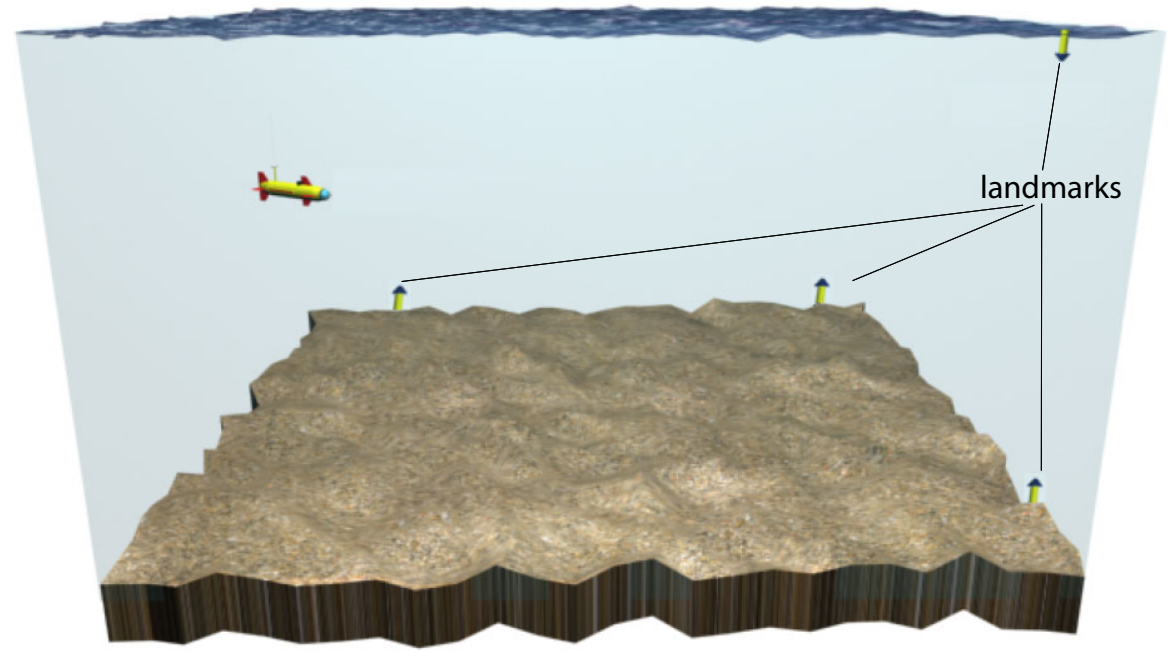

Fig. 1. Mission scenario. 


$$
\mathbf{a}(t)=\dot{\mathbf{v}}(t)+\mathbf{S}(\boldsymbol{\omega}(t)) \mathbf{v}(t)-\mathbf{g}(t)
$$

where $\mathbf{g}(t) \in \mathbb{R}^{3}$ denotes the acceleration of gravity, expressed in body-fixed coordinates. Ideal accelerometers would not measure the gravitational term but in practice this term must be considered due to the inherent physics of the accelerometers, see [32] for further details. Since the magnitude of $\mathbf{g}(t)$ is usually well known, it would be possible to cancel this term in (2) as the attitude of the vehicle is measured. However, even small errors on $\mathbf{R}(t)$ would lead to large errors in the acceleration compensation. Therefore, the acceleration of gravity is considered here as unknown state, in addition to $\mathbf{p}(t)$ and $\mathbf{v}(t)$.

Finally, let $\mathbf{s}_{i} \in \mathbb{R}^{3}, i=1, \ldots, n_{L}$, denote the inertial positions of the landmarks. Then, the range measurements are given by

$$
r_{i}(t)=\left\|\mathbf{s}_{i}-\mathbf{p}(t)\right\| .
$$

The derivative of the velocity can be written, from (2), as

$$
\dot{\mathbf{v}}(t)=\mathbf{a}(t)-\mathbf{S}(\boldsymbol{\omega}(t)) \mathbf{v}(t)+\mathbf{g}(t) .
$$

The derivative of $\mathbf{g}(t)$, assuming that the acceleration of gravity is locally constant in inertial coordinates, is given by

$$
\dot{\mathbf{g}}(t)=-\mathbf{S}(\boldsymbol{\omega}(t)) \mathbf{g}(t) .
$$

Combining (1) and (3)-(5) yields the nonlinear system

$$
\left\{\begin{array}{l}
\dot{\mathbf{p}}(t)=\mathbf{R}(t) \mathbf{v}(t) \\
\dot{\mathbf{v}}(t)=\mathbf{a}(t)-\mathbf{S}(\boldsymbol{\omega}(t)) \mathbf{v}(t)+\mathbf{g}(t) \\
\dot{\mathbf{g}}(t)=-\mathbf{S}(\boldsymbol{\omega}(t)) \mathbf{g}(t) \\
r_{1}(t)=\left\|\mathbf{s}_{1}-\mathbf{p}(t)\right\| \\
\vdots \\
r_{n_{L}}(t)=\left\|\mathbf{s}_{n_{L}}-\mathbf{p}(t)\right\|
\end{array} .\right.
$$

The problem considered in the paper is the design of an estimator for (6).

\subsection{Long baseline configuration}

Long baseline acoustic configurations have been widely used in the past in the design of navigation systems. LBL systems owe their name to the property that the distance between the baseline transponders is long or similar to the distance between the vehicle and the transponders. This is in contrast with USBL systems, where the distance between the transponder and the vehicle is much larger than the distance between receivers of the USBL system.
In the remainder of the paper, and for the long baseline configuration, the following assumption is considered:

Assumption 1. There exist at least four noncoplanar landmarks.

When there exist at least four noncoplanar landmarks, it is always possible to determine the position of the vehicle from the range measurements and therefore it is expected that the system is observable. When there are fewer measurements that is not always true. The cases of two and three range measurements are considered in Section V, while preliminary work on the case of single range readings can be found in $[28,29]$.

\section{FILTER DESIGN}

This section presents the main results of the paper. Firstly, a state transformation is applied, in Section 3.1, to reduce the complexity of the system dynamics. Afterwards, state augmentation is proposed, in Section 3.2, in order to derive sensor-based deterministic system dynamics, and in Section 3.3 the observability of the resulting system is analyzed. The design of a Kalman filter is detailed in Section 3.4.

\subsection{State transformation}

Let $\mathbf{T}(t):=\operatorname{diag}(\mathbf{I}, \mathbf{R}(t), \mathbf{R}(t)) \in \mathbb{R}^{9 \times 9}$ and consider the Lyapunov state transformation

$$
\left[\begin{array}{l}
\mathbf{x}_{1}(t) \\
\mathbf{x}_{2}(t) \\
\mathbf{x}_{3}(t)
\end{array}\right]:=\mathbf{T}(t)\left[\begin{array}{l}
\mathbf{p}(t) \\
\mathbf{v}(t) \\
\mathbf{g}(t)
\end{array}\right] .
$$

The new system dynamics are given by

$$
\left\{\begin{array}{l}
\dot{\mathbf{x}}_{1}(t)=\mathbf{x}_{2}(t) \\
\dot{\mathbf{x}}_{2}(t)=\mathbf{x}_{3}(t)+\mathbf{u}(t) \\
\dot{\mathbf{x}}_{3}(t)=\mathbf{0} \\
r_{1}(t)=\left\|\mathbf{s}_{1}-\mathbf{x}_{1}(t)\right\| \\
\vdots \\
r_{n_{L}}(t)=\left\|\mathbf{s}_{n_{L}}-\mathbf{x}_{1}(t)\right\|
\end{array},\right.
$$

where $\mathbf{u}(t):=\mathbf{R}(t) \mathbf{a}(t)$. The advantage of considering the state transformation (7) is that the new system is time invariant, even though it is still nonlinear.

Remark 1. The state transformation (7) corresponds to expressing the vehicle navigation state in inertial coordinates. While the problem could have been originally formulated in inertial coordinates, it was formulated instead in the most 
appropriate space, i.e., the space that corresponds to the sensor measurements, therefore avoiding acceleration cancellation problems, as discussed in [13]. The state transformation is here applied only for theoretical purposes, as it simplifies the computations. However, the final filter design will be obtained in the original coordinate space, as detailed in Section 3.4.

\subsection{State augmentation}

To derive a system that can be regarded as linear and that mimics the dynamics of the nonlinear system (8), define $n_{L}+4$ additional scalar state variables as

$$
\left\{\begin{array}{l}
x_{4}(t):=r_{1}(t) \\
\vdots \\
x_{n_{L}+3}(t):=r_{n_{L}}(t) \\
x_{n_{L}+4}(t):=\mathbf{x}_{1}(t) \cdot \mathbf{x}_{2}(t) \\
x_{n_{L}+5}(t):=\mathbf{x}_{1}(t) \cdot \mathbf{x}_{3}(t)+\left\|\mathbf{x}_{2}(t)\right\|^{2} \\
x_{n_{L}+6}:=\mathbf{x}_{2}(t) \cdot \mathbf{x}_{3}(t) \\
x_{n_{L}+7}(t):=\left\|\mathbf{x}_{3}(t)\right\|^{2}
\end{array}\right.
$$

and let $(t) \in \mathbb{R}^{n}$,

$$
\boldsymbol{x}(t)=\left[\begin{array}{llllll}
\mathbf{x}_{1}^{T}(t) & \mathbf{x}_{2}^{T}(t) & \mathbf{x}_{3}^{T}(t) & x_{4}(t) & \ldots & x_{n_{L}+7}(t)
\end{array}\right]^{T}
$$

$n=13+n_{L}$, be the augmented state. It is easy to verify that the augmented state dynamics can be written as $\dot{x}(t)=\mathbf{A}(t) x(t)+\mathbf{B u}(t)$, where

$$
\mathbf{A}(t)=\left[\begin{array}{cccccccccc}
\mathbf{0} & \mathbf{I} & \mathbf{0} & \mathbf{0} & \ldots & \mathbf{0} & \mathbf{0} & \mathbf{0} & \mathbf{0} & \mathbf{0} \\
\mathbf{0} & \mathbf{0} & \mathbf{I} & \mathbf{0} & \ldots & \mathbf{0} & \mathbf{0} & \mathbf{0} & \mathbf{0} & \mathbf{0} \\
\mathbf{0} & \mathbf{0} & \mathbf{0} & \mathbf{0} & \ldots & \mathbf{0} & \mathbf{0} & \mathbf{0} & \mathbf{0} & \mathbf{0} \\
\mathbf{0} & -\frac{\mathbf{s}_{1}^{T}}{r_{1}(t)} & \mathbf{0} & 0 & \ldots & 0 & \frac{1}{r_{1}(t)} & 0 & 0 & 0 \\
\vdots & \vdots & \vdots & \vdots & & \vdots & \vdots & \vdots & \vdots & \vdots \\
\mathbf{0} & -\frac{\mathbf{s}_{n_{L}}^{T}}{r_{n_{L}}(t)} & \mathbf{0} & 0 & \ldots & 0 & \frac{1}{r_{n_{L}}(t)} & 0 & 0 & 0 \\
\mathbf{u}^{T}(t) & \mathbf{0} & \mathbf{0} & 0 & \ldots & 0 & 0 & 1 & 0 & 0 \\
\mathbf{0} & 2 \mathbf{u}^{T}(t) & \mathbf{0} & 0 & \ldots & 0 & 0 & 0 & 3 & 0 \\
\mathbf{0} & \mathbf{0} & \mathbf{u}^{T}(t) & 0 & \ldots & 0 & 0 & 0 & 0 & 1 \\
\mathbf{0} & \mathbf{0} & \mathbf{0} & 0 & \ldots & 0 & 0 & 0 & 0 & 0
\end{array}\right]
$$

and $\mathbf{B}=\left[\begin{array}{lll}\mathbf{0 I 0} 0 & \ldots & 0\end{array}\right]^{T}$.

Remark 2. The first $n_{L}$ additional states correspond to the range measurements. As these are measured, it is now possible to also consider a linear output. The remaining additional four states correspond to nonlinear terms of the derivatives of the range readings. In this way, it is possible to express the derivatives of the range measurements as a linear combination of the states, as will be shown.

In order to complete the augmented system dynamics, notice first that the states $x_{4}(t), \ldots, x_{n_{L}+3}(t)$ are measured. In addition to that, it can be shown that

$$
\frac{2\left(\mathbf{s}_{i}-\mathbf{s}_{j}\right) \cdot \mathbf{x}_{1}(t)}{r_{i}(t)+r_{j}(t)}+x_{3+i}(t)-x_{3+j}(t)=\frac{\left\|\mathbf{s}_{i}\right\|^{2}-\left\|\mathbf{s}_{j}\right\|^{2}}{r_{i}(t)+r_{j}(t)},
$$

$i, j \in\left\{1, \ldots, n_{L}\right\}, i \neq j$, where the right side of (10) is available and the left side depends on the system state. Discarding the original nonlinear system output, it is possible to write an augmented system output as

$$
\left\{\begin{array}{l}
y_{1}(t)=x_{4}(t) \\
\vdots \\
y_{n_{L}}(t)=x_{3+n_{L}}(t) \\
y_{n_{L}+1}(t)=\frac{2\left(\mathbf{s}_{1}-\mathbf{s}_{2}\right) \cdot \mathbf{x}_{1}(t)}{r_{1}(t)+r_{2}(t)}+x_{3+1}(t)-x_{3+2}(t) \\
y_{n_{L}+2}(t)=\frac{2\left(\mathbf{s}_{1}-\mathbf{s}_{3}\right) \cdot \mathbf{x}_{1}(t)}{r_{1}(t)+r_{3}(t)}+x_{3+1}(t)-x_{3+3}(t) \\
\vdots \\
y_{n_{L}+C_{2}^{n L}-1}(t)=\frac{2\left(\mathbf{s}_{n_{L}-2}-\mathbf{s}_{n_{L}}\right) \cdot \mathbf{x}_{1}(t)}{r_{n_{L}-2}(t)+r_{n_{L}}(t)}+x_{n_{L}+3-2}-x_{n_{L}+3}(t) \\
y_{n_{L}+C_{2}^{n L}}(t)=\frac{2\left(\mathbf{s}_{n_{L}-1}-\mathbf{s}_{n_{L}}\right) \cdot \mathbf{x}_{1}(t)}{r_{n_{L}-1}(t)+r_{n_{L}}(t)}+x_{n_{L}+3-1}-x_{n_{L}+3}(t)
\end{array},\right.
$$

where $C_{2}^{n_{L}}$ is the number of 2-combinations of $n_{L}$ elements, i.e. $C_{2}^{n_{L}}=n_{L}\left(n_{L}-1\right) / 2$.

Remark 3. The first $n_{L}$ outputs correspond simply to the range measurements. The remaining terms are feeding the system with information about the long baseline structure of the transponders.

In compact form, the augmented system dynamics are given by

$$
\left\{\begin{array}{l}
\dot{\boldsymbol{x}}(t)=\mathbf{A}(t) \boldsymbol{x}(t)+\mathbf{B u}(t) \\
\mathbf{y}(t)=\mathbf{C}(t) \boldsymbol{x}(t)
\end{array}\right.
$$

where

$$
\mathbf{C}(t)=\left[\begin{array}{ccccc}
\mathbf{0}_{n_{L} \times 3} & \mathbf{0}_{n_{L} \times 3} & \mathbf{0}_{n_{L} \times 3} & \mathbf{I}_{n_{L}} & \mathbf{0}_{n_{L} \times 4} \\
\hline \mathbf{C}_{1}(t) & \mathbf{0}_{C_{2}^{n L} \times 3} & \mathbf{0}_{C_{2}^{n L} \times 3} & \mathbf{C}_{2} & \mathbf{0}_{C_{2}^{n_{L} \times 4}}
\end{array}\right],
$$




$$
\mathbf{C}_{1}(t)=\left[\begin{array}{c}
\frac{2\left(\mathbf{s}_{1}-\mathbf{s}_{2}\right)^{T}}{r_{1}(t)+r_{2}(t)} \\
\frac{2\left(\mathbf{s}_{1}-\mathbf{s}_{3}\right)^{T}}{r_{1}(t)+r_{3}(t)} \\
\vdots \\
\frac{2\left(\mathbf{s}_{n_{L}-2}-\mathbf{s}_{n_{L}}\right)^{T}}{r_{n_{L}-2}(t)+r_{n_{L}}(t)} \\
\frac{2\left(\mathbf{s}_{n_{L}-1}-\mathbf{s}_{n_{L}}\right)^{T}}{r_{n_{L}-1}(t)+r_{n_{L}}(t)}
\end{array}\right] \in \mathbb{R}^{C_{2}^{n_{L} \times 3},}
$$

and

$$
\mathbf{C}_{2}=\left[\begin{array}{ccccccc}
1 & -1 & 0 & \ldots & \ldots & \ldots & 0 \\
1 & 0 & -1 & 0 & \ldots & \ldots & 0 \\
& & & \vdots & & & \\
0 & \ldots & \ldots & 0 & 1 & 0 & -1 \\
0 & \ldots & \ldots & \ldots & 0 & 1 & -1
\end{array}\right] \in \mathbb{R}^{C_{2}^{n L} \times n_{L}}
$$

is the matrix that encodes all the combinations of differences of pairs of ranges.

The dynamic system (11) can be regarded as a linear time-varying system, even though the system matrices $\mathbf{A}(t)$ and $\mathbf{C}(t)$ depend explicitly on the system input and output, as evidenced by (9) and (12). Nevertheless, this is not a problem for observability purposes as both the input and output of the system are available as continuous bounded signals. This just suggests, in this case, that the observability of (11) may be connected with the evolution of the system input, output, or both, which is not common and does not happen when this matrix does not depend on the system input or output. The underlying reason for this is that the system is, in fact, nonlinear, even though it admits a linear interpretation. In the specific case addressed in this section it will be shown that the system is observable, regardless of the input and output. When only two or three range measurements are available, that is not the case and additional conditions are required. This will be detailed in Section V.

Before proceeding with the observability analysis of (11), notice that there is nothing in (11) imposing

$$
\left\{\begin{array}{l}
r_{1}(t)=\left\|\mathbf{s}_{1}-\mathbf{x}_{1}(t)\right\| \\
\vdots \\
r_{n_{L}}(t)=\left\|\mathbf{s}_{n_{L}}-\mathbf{x}_{1}(t)\right\| \\
x_{n_{L}+4}(t)=\mathbf{x}_{1}(t) \cdot \mathbf{x}_{2}(t) \\
x_{n_{L}+5}(t)=\mathbf{x}_{1}(t) \cdot \mathbf{x}_{3}(t)+\left\|\mathbf{x}_{2}(t)\right\|^{2} \\
x_{n_{L}+6}(t)=\mathbf{x}_{2}(t) \cdot \mathbf{x}_{3}(t) \\
x_{n_{L}+7}(t)=\left\|\mathbf{x}_{3}(t)\right\|^{2}
\end{array} .\right.
$$

Although these restrictions could be easily imposed including artificial outputs, this form is preferred since it allows us to regard the system as linear. However, care must be taken when extrapolating conclusions from the observability of (11) to the observability of (8) or (6).

Finally, the following assumption is required so that (9) is well defined.

Assumption 2. The motion of the vehicle is such that

$$
\underset{R_{m}>0}{\exists} \underset{t \geq t_{0}}{\forall} r_{i}(t) \geq R_{m}
$$

for all $i=1, \ldots, n_{L}$.

This is not restrictive from the practical point of view since it makes no sense to have the vehicle at the same position of a landmark, where an acoustic transponder is installed.

Remark 4. It is actually possible to derive all the results presented in the paper without Assumption 2, considering the square of the range instead of the range. This form is preferred since it offers, in practice, better performance for large distances when considering sensor noise, as the noise could be amplified and loose the good zero mean and Gaussian characteristics with the square operation.

Remark 5. Even though all combinations of pairs were considered in the definition of the augmented output, in general it is not necessary to consider them all. Indeed, all results presented hereafter hold as long as $\mathbf{C}_{1}(t)$ has maximum rank. For the sake of completeness, all combinations are considered in the paper, without any loss of generality.

\subsection{Observability analysis}

In the previous section an augmented nonlinear system that can be regarded as LTV was derived so as to capture the behavior of the original nonlinear system. The analysis of this augmented system is carried out in this section and its behavior compared with that of the original nonlinear system. First, the following lemma is presented, which addresses the problem of observability of systems like (11) where the system matrices may depend on the system input, output, or both.

Lemma 1. Consider the nonlinear system

$$
\left\{\begin{array}{l}
\dot{\mathbf{x}}(t)=\mathcal{A}(t, \mathbf{u}(t), \mathbf{y}(t)) \mathbf{x}(t)+\mathcal{B}(t) \mathbf{u}(t) \\
\mathbf{y}(t)=\mathcal{C}(t, \mathbf{u}(t), \mathbf{y}(t)) \mathbf{x}(t)
\end{array}\right.
$$

If the observability Gramian $\mathcal{W}\left(t_{0}, t_{f}\right)$ associated with the pair $(\mathcal{A}(t, \mathbf{u}(t), \mathbf{y}(t)), \mathcal{C}(t, \mathbf{u}(t), \mathbf{y}(t))) \quad$ on $\quad \mathcal{I}=\left[t_{0}, t_{f}\right] \quad$ is invertible, then the nonlinear system (13) is observable on $\mathcal{I}$ in the sense that any initial condition is uniquely determined by the corresponding response $\{\mathbf{y}(t), t \in \mathcal{I}\}$ and system input $\{\mathbf{u}(t), t \in \mathcal{I}\}$. 
Proof. This lemma is very similar to [30, Lemma 1], the only difference being in the dependence here of the system matrix $\mathcal{C}$ on $\mathbf{u}(t)$ and $\mathbf{y}(t)$, in addition to $t$. However, the proof stands as these quantities are all available for observability purposes.

The following theorem establishes the observability of the nonlinear system (11).

Theorem 1. Suppose that Assumptions 1 and 2 are verified. Then, the nonlinear system (11) is observable on $\left[t_{0}, t_{f}\right], t_{0}<t_{f}$, in the sense that any initial condition is uniquely determined by the corresponding response $\left\{\mathbf{y}(t), t \in\left[t_{0}, t_{f}\right]\right\}$ and the system input $\left\{\mathbf{u}(t), t \in\left[t_{0}, t_{f}\right]\right\}$.

Proof. The proof follows by contradiction. Suppose that the nonlinear system (11) is not observable on $\mathcal{I}:=\left[t_{0}, t_{f}\right]$ in the sense established in Lemma 1. Then, it follows from Lemma 1 that there exists a non-null vector $\mathbf{d} \in \mathbb{R}^{13+n_{L}}$,

$$
\mathbf{d}=\left[\begin{array}{llllllll}
\mathbf{d}_{1}^{T} & \mathbf{d}_{2}^{T} & \mathbf{d}_{3}^{T} & \mathbf{d}_{4}^{T} & d_{5} & d_{6} & d_{7} & d_{8}
\end{array}\right]^{T},
$$

$\mathbf{d}_{1}, \mathbf{d}_{2}, \mathbf{d}_{3} \in \mathbb{R}^{3}, \mathbf{d}_{4} \in \mathbb{R}^{n_{L}}, d_{5}, \ldots, d_{8} \in \mathbb{R}$, such that

$$
\mathbf{d}^{T} \mathcal{W}\left(t_{0}, t\right) \mathbf{d}=0
$$

for all $t \in \mathcal{I}$, where $W\left(t_{0}, t_{f}\right)$ denotes the observability Gramian associated with the pair $(\mathbf{A}(t), \mathbf{C}(t))$. The dependence of $\mathbf{A}(t)$ and $\mathbf{C}(t)$ on the input and output is omitted here for sake of readability. By definition, the observability Gramian is given by

$$
\mathcal{W}\left(t_{0}, t_{f}\right)=\int_{t_{0}}^{t_{f}} \phi^{T}\left(t, t_{0}\right) \mathbf{C}^{T}(t) \mathbf{C}(t) \phi\left(t, t_{0}\right) d t
$$

where $\phi\left(t, t_{0}\right)$ stands for the transition matrix associated with $\mathbf{A}(t)$, presented in Appendix A. Substituting (15) in (14) immediately yields

$$
\int_{t_{0}}^{t}\left\|\mathbf{C}(\tau) \phi\left(\tau, t_{0}\right) \mathbf{d}\right\|^{2} d \tau=0
$$

for all $t \in \mathcal{I}$. Taking the time derivative of (16) yields $\left\|\mathbf{C}(t) \phi\left(t, t_{0}\right) \mathbf{d}\right\|^{2}=0$, which implies, in particular, that

$$
\mathbf{C}(t) \phi\left(t, t_{0}\right) \mathbf{d}=0
$$

for all $t \in \mathcal{I}$. Substituting $t=t_{0}$ in (17) gives

$$
\left[\begin{array}{c}
\mathbf{d}_{4} \\
\mathbf{C}_{1}\left(t_{0}\right) \mathbf{d}_{1}+\mathbf{C}_{2} \mathbf{d}_{4}
\end{array}\right]=\mathbf{0}
$$

which implies immediately that

$$
\mathbf{d}_{4}=\mathbf{0} .
$$

Substituting (19) in (18) gives

$$
\left[\begin{array}{c}
\frac{2}{r_{1}\left(t_{0}\right)+r_{2}\left(t_{0}\right)}\left(\mathbf{s}_{1}-\mathbf{s}_{2}\right)^{T} \\
\frac{2}{r_{1}\left(t_{0}\right)+r_{3}\left(t_{0}\right)}\left(\mathbf{s}_{1}-\mathbf{s}_{3}\right)^{T} \\
\vdots \\
\frac{2}{r_{n_{L}-2}\left(t_{0}\right)+r_{n_{L}}\left(t_{0}\right)}\left(\mathbf{s}_{n_{L}-2}-\mathbf{s}_{n_{L}}\right)^{T} \\
\frac{2}{r_{n_{L}-1}\left(t_{0}\right)+r_{n_{L}}\left(t_{0}\right)}\left(\mathbf{s}_{n_{L}-1}-\mathbf{s}_{n_{L}}\right)^{T}
\end{array}\right] \mathbf{d}_{1}=\mathbf{0} .
$$

It is straightforward to show that, under Assumption 1, the only solution of (20) is

$$
\mathbf{d}_{1}=\mathbf{0} \text {. }
$$

Now, from (17), it is possible to write

$$
\frac{d}{d t}\left[\mathbf{C}(t) \phi\left(t, t_{0}\right) \mathbf{d}\right]=\mathbf{0}
$$

for all $t \in \mathcal{I}$. Let

$$
\mathbf{u}^{[1]}\left(t, t_{0}\right):=\int_{t_{0}}^{t} \mathbf{u}(\sigma) d \sigma
$$

and

$$
\mathbf{u}^{[2]}\left(t, t_{0}\right):=\int_{t_{0}}^{t} \int_{t_{0}}^{\sigma_{1}} \mathbf{u}\left(\sigma_{2}\right) d \sigma_{2} d \sigma_{1}
$$

Expanding (22), and considering (19) and (21), allows us to write

$$
\begin{aligned}
& \frac{\left[-\mathbf{s}_{i}+\left(t-t_{0}\right) \mathbf{u}^{[1]}\left(t, t_{0}\right)+\mathbf{u}^{[2]}\left(t, t_{0}\right)\right]^{T} \mathbf{d}_{2}}{r_{i}(t)} \\
& -\left(t-t_{0}\right) \frac{\left[\mathbf{s}_{i}-\frac{t-t_{0}}{2} \mathbf{u}^{[1]}\left(t, t_{0}\right)-\mathbf{u}^{[2]}\left(t, t_{0}\right)\right]^{T} \mathbf{d}_{3}}{r_{i}(t)}+\frac{1}{r_{i}(t)} d_{5} \\
& +\frac{t-t_{0}}{r_{i}(t)} d_{6}+\frac{3}{2} \frac{\left(t-t_{0}\right)^{2}}{r_{i}(t)} d_{7}+\frac{1}{2} \frac{\left(t-t_{0}\right)^{3}}{r_{i}(t)} d_{8}=0
\end{aligned}
$$


for all $i=1, \ldots, n_{L}, t \in I$ or, equivalently,

$$
\begin{aligned}
& {\left[-\mathbf{s}_{i}+\left(t-t_{0}\right) \mathbf{u}^{[1]}\left(t, t_{0}\right)+\mathbf{u}^{[2]}\left(t, t_{0}\right)\right]^{T} \mathbf{d}_{2}} \\
& \quad-\left(t-t_{0}\right)\left[\mathbf{s}_{i}-\frac{t-t_{0}}{2} \mathbf{u}^{[1]}\left(t, t_{0}\right)-\mathbf{u}^{[2]}\left(t, t_{0}\right)\right]^{T} \mathbf{d}_{3} \\
& \quad+d_{5}+\left(t-t_{0}\right) d_{6}+\frac{3}{2}\left(t-t_{0}\right)^{2} d_{7} \\
& \quad+\frac{1}{2}\left(t-t_{0}\right)^{3} d_{8}=0 .
\end{aligned}
$$

Substituting $t=t_{0}$ in (23) gives

$$
\left[\begin{array}{cc}
-\mathbf{s}_{1}^{T} & 1 \\
-\mathbf{s}_{2}^{T} & 1 \\
\vdots & \\
-\mathbf{s}_{n_{L}-1}^{T} & 1 \\
-\mathbf{s}_{n_{L}}^{T} & 1
\end{array}\right]\left[\begin{array}{l}
\mathbf{d}_{2} \\
d_{5}
\end{array}\right]=\mathbf{0} .
$$

Again, it can be shown that, under Assumption 1, the only solution of (24) is

$$
\left\{\begin{array}{l}
\mathbf{d}_{2}=\mathbf{0} \\
d_{5}=0
\end{array}\right. \text {. }
$$

Now, considering (25) in (23) and taking its time derivative gives

$$
\begin{gathered}
-\left[\mathbf{s}_{i}-\frac{\left(t-t_{0}\right)^{2}}{2} \mathbf{u}(t)-\left(t-t_{0}\right) \mathbf{u}^{[1]}\left(t, t_{0}\right)\right]^{T} \mathbf{d}_{3} \\
+\left[\left(t-t_{0}\right) \mathbf{u}^{[1]}\left(t, t_{0}\right)+\mathbf{u}^{[2]}\left(t, t_{0}\right)\right]^{T} \mathbf{d}_{3} \\
\quad+d_{6}+3\left(t-t_{0}\right) d_{7}+\frac{3}{2}\left(t-t_{0}\right)^{2} d_{8}=0
\end{gathered}
$$

for all $i=1, \ldots, n_{L}$ and $t \in I$. Setting $t=t_{0}$ in (26) gives

$$
\left[\begin{array}{cc}
-\mathbf{s}_{1}^{T} & 1 \\
-\mathbf{s}_{2}^{T} & 1 \\
\vdots & \\
-\mathbf{s}_{n_{L}-1}^{T} & 1 \\
-\mathbf{s}_{n_{L}}^{T} & 1
\end{array}\right]\left[\begin{array}{l}
\mathbf{d}_{3} \\
d_{6}
\end{array}\right]=\mathbf{0} .
$$

Again, under Assumption 1, the only solution of (27) is

$$
\left\{\begin{array}{l}
\mathbf{d}_{3}=\mathbf{0} \\
d_{6}=0
\end{array} .\right.
$$

Finally, substituting (28) in (26) gives

$$
3\left(t-t_{0}\right) d_{7}+\frac{3}{2}\left(t-t_{0}\right)^{2} d_{8}=0
$$

for all $t \in I$. Since $t-t_{0}$ and $\left(t-t_{0}\right)^{2}$ are linearly independent functions, the only solution of (29) is $d_{7}=d_{8}=0$. But that contradicts the hypothesis of existence of a non-null vector d such that (14) is true. Therefore, (11) is observable.

Next, the observability of (8) is analyzed, building on the observability results of Theorem 1 . Notice that, in the usual definition of observability for nonlinear systems, it is not necessarily true that every admissible input distinguishes between initial conditions [33]. However, that is implied in the following theorem.

Theorem 2. Suppose that Assumptions 1 and 2 are verified. Then, the nonlinear system (8) is observable on $\left[t_{0}, t_{f}\right]$ in the sense that any initial condition is uniquely determined by the corresponding response $\left\{\mathbf{y}(t), t \in\left[t_{0}, t_{f}\right]\right\}$ and the system input $\left\{\mathbf{u}(t), t \in\left[t_{0}, t_{f}\right]\right\}$. Moreover, a state observer for (11) with globally exponentially stable error dynamics is also a state observer for the nonlinear system (8), with globally exponentially stable error dynamics.

Proof. From Theorem 1 it is possible to say that, given the system response $\left\{\mathbf{y}(t), t \in\left[t_{0}, t_{f}\right]\right\}$ and the system input $\left\{\mathbf{u}(t), t \in\left[t_{0}, t_{f}\right]\right\}$, the initial state of (11) is uniquely defined. Let

$$
\mathbf{z}\left(t_{0}\right)=\left[\begin{array}{c}
\mathbf{z}_{1}\left(t_{0}\right) \\
\mathbf{z}_{2}\left(t_{0}\right) \\
\mathbf{z}_{3}\left(t_{0}\right) \\
z_{4}\left(t_{0}\right) \\
\vdots \\
z_{n_{L}+7}\left(t_{0}\right)
\end{array}\right] \in \mathbb{R}^{13+n_{L}},
$$

$\mathbf{z}_{1}\left(t_{0}\right), \mathbf{z}_{2}\left(t_{0}\right), \mathbf{z}_{3}\left(t_{0}\right) \in \mathbb{R}^{3}, z_{4}, \ldots, z_{n_{L}+7}\left(t_{0}\right) \in \mathbb{R}$, be the initial state of (11) and

$$
\mathbf{x}\left(t_{0}\right)=\left[\begin{array}{l}
\mathbf{x}_{1}\left(t_{0}\right) \\
\mathbf{x}_{2}\left(t_{0}\right) \\
\mathbf{x}_{3}\left(t_{0}\right)
\end{array}\right], \mathbf{x}_{1}\left(t_{0}\right), \mathbf{x}_{2}\left(t_{0}\right), \mathbf{x}_{3}\left(t_{0}\right) \in \mathbb{R}^{3},
$$

be the initial state of the nonlinear system (8). Notice first that, as $z_{3+i}\left(t_{0}\right), i=1, \ldots, n_{L}$, correspond to actual outputs of the system, the ranges, it immediately follows that

$$
z_{3+i}\left(t_{0}\right)=r_{i}\left(t_{0}\right), i=1, \ldots, n_{L} .
$$

Evaluating the outputs of (11) $y_{n_{L}+1}(t)$ to $y_{n_{L}+C_{2}^{n_{L}}}(t)$ at $t=t_{0}$ allows us to write

$$
\frac{2\left(\mathbf{s}_{i}-\mathbf{s}_{j}\right) \cdot \mathbf{z}_{1}\left(t_{0}\right)}{r_{i}\left(t_{0}\right)+r_{j}\left(t_{0}\right)}+z_{3+i}\left(t_{0}\right)-z_{3+j}\left(t_{0}\right)=\frac{\left\|\mathbf{s}_{i}\right\|^{2}-\left\|\mathbf{s}_{j}\right\|^{2}}{r_{i}\left(t_{0}\right)+r_{j}\left(t_{0}\right)}
$$

or, equivalently, using (30),

$$
2\left(\mathbf{s}_{i}-\mathbf{s}_{j}\right) \cdot \mathbf{z}_{1}\left(t_{0}\right)=\left\|\mathbf{s}_{i}\right\|^{2}-\left\|\mathbf{s}_{j}\right\|^{2}-\left[r_{i}^{2}\left(t_{0}\right)-r_{j}^{2}\left(t_{0}\right)\right],
$$


for all $i, j \in\left\{1, \ldots, n_{L}\right\}, i \neq j$. Substituting (3) in (31) and as $\mathbf{x}_{1}(t)=\mathbf{p}(t)$, it follows that $\left(\mathbf{s}_{i}-\mathbf{s}_{j}\right) \cdot\left[\mathbf{x}_{1}\left(t_{0}\right)-\mathbf{z}_{1}\left(t_{0}\right)\right]=0$ for all $i, j \in\left\{1, \ldots, n_{L}\right\}, i \neq j$, or, in compact form,

$$
\left[\begin{array}{c}
\left(\mathbf{s}_{1}-\mathbf{s}_{2}\right)^{T} \\
\left(\mathbf{s}_{1}-\mathbf{s}_{3}\right)^{T} \\
\vdots \\
\left(\mathbf{s}_{n_{L}-2}-\mathbf{s}_{n_{L}}\right)^{T} \\
\left(\mathbf{s}_{n_{L}-1}-\mathbf{s}_{n_{L}}\right)^{T}
\end{array}\right]\left[\mathbf{x}_{1}\left(t_{0}\right)-\mathbf{z}_{1}\left(t_{0}\right)\right]=\mathbf{0} .
$$

Under Assumption 1 the only solution of (32) is

$$
\mathbf{x}_{1}\left(t_{0}\right)=\mathbf{z}_{1}\left(t_{0}\right) .
$$

The evolution, for the nonlinear system, of $\mathbf{x}_{1}(t)$, is given by

$\mathbf{x}_{1}(t)=\mathbf{x}_{1}\left(t_{0}\right)+\left(t-t_{0}\right) \mathbf{x}_{2}\left(t_{0}\right)+\frac{\left(t-t_{0}\right)^{2}}{2} \mathbf{x}_{3}\left(t_{0}\right)+\mathbf{u}^{[2]}\left(t, t_{0}\right)$

and therefore the output of the nonlinear system satisfies

$$
\begin{aligned}
r_{i}^{2}(t)= & \left\|\mathbf{x}_{1}\left(t_{0}\right)-\mathbf{s}_{i}\right\|^{2}+\left(t-t_{0}\right)^{2}\left\|\mathbf{x}_{2}\left(t_{0}\right)\right\|^{2}+\frac{\left(t-t_{0}\right)^{4}}{4}\left\|\mathbf{x}_{3}\left(t_{0}\right)\right\|^{2} \\
& +\left\|\mathbf{u}^{[2]}\left(t, t_{0}\right)\right\|^{2}+2\left(t-t_{0}\right) \mathbf{x}_{1}\left(t_{0}\right) \cdot \mathbf{x}_{2}\left(t_{0}\right) \\
& +\left(t-t_{0}\right)^{2} \mathbf{x}_{1}\left(t_{0}\right) \cdot \mathbf{x}_{3}\left(t_{0}\right)+\left(t-t_{0}\right)^{3} \mathbf{x}_{2}\left(t_{0}\right) \cdot \mathbf{x}_{3}\left(t_{0}\right) \\
& -2\left(t-t_{0}\right) \mathbf{s}_{i} \cdot \mathbf{x}_{2}\left(t_{0}\right)-\left(t-t_{0}\right)^{2} \mathbf{s}_{i} \cdot \mathbf{x}_{3}\left(t_{0}\right) \\
& -2 \mathbf{s}_{i} \cdot \mathbf{u}^{[2]}\left(t, t_{0}\right)+2 \mathbf{x}_{1}\left(t_{0}\right) \cdot \mathbf{u}^{[2]}\left(t, t_{0}\right) \\
& +2 \mathbf{x}_{2}\left(t_{0}\right) \cdot\left(t-t_{0}\right) \mathbf{u}^{[2]}\left(t, t_{0}\right) \\
& +\mathbf{x}_{3}\left(t_{0}\right) \cdot\left(t-t_{0}\right)^{2} \mathbf{u}^{[2]}\left(t, t_{0}\right) .
\end{aligned}
$$

From (35) it is possible to write

$$
\begin{aligned}
r_{i}^{2}(t)-r_{j}^{2}(t)= & \left\|\mathbf{x}_{1}\left(t_{0}\right)-\mathbf{s}_{i}\right\|^{2}-\left\|\mathbf{x}_{1}\left(t_{0}\right)-\mathbf{s}_{j}\right\|^{2} \\
& -2\left(\mathbf{s}_{i}-\mathbf{s}_{j}\right) \cdot \mathbf{u}^{[2]}\left(t, t_{0}\right) \\
& -2\left(t-t_{0}\right)\left(\mathbf{s}_{i}-\mathbf{s}_{j}\right) \cdot \mathbf{x}_{2}\left(t_{0}\right) \\
& -\left(t-t_{0}\right)^{2}\left(\mathbf{s}_{i}-\mathbf{s}_{j}\right) \cdot \mathbf{x}_{3}\left(t_{0}\right)
\end{aligned}
$$

for all $i, j \in\left\{1, \ldots, n_{L}\right\}$ and $t \in \mathcal{I}$. On the other hand, it is possible to write the evolution of the square of the range readings as a function of the initial state of (11) as

$$
\begin{aligned}
r_{i}^{2}(t)= & 2\left[\mathbf{z}_{1}\left(t_{0}\right)-\mathbf{s}_{i}\right] \cdot \mathbf{u}^{[2]}\left(t, t_{0}\right) \\
& +2\left(t-t_{0}\right)\left[\mathbf{u}^{[2]}\left(t, t_{0}\right)-\mathbf{s}_{i}\right] \cdot \mathbf{z}_{2}\left(t_{0}\right) \\
& +\left(t-t_{0}\right)^{2}\left[\mathbf{u}^{[2]}\left(t, t_{0}\right)-\mathbf{s}_{i}\right] \cdot \mathbf{z}_{3}\left(t_{0}\right) \\
& +z_{3+i}^{2}\left(t_{0}\right)+2\left(t-t_{0}\right) z_{n_{L}+4}\left(t_{0}\right) \\
& +\left(t-t_{0}\right)^{2} z_{n_{L}+5}\left(t_{0}\right)+\left(t-t_{0}\right)^{3} z_{n_{L}+6}\left(t_{0}\right) \\
& +\frac{\left(t-t_{0}\right)^{4}}{4} z_{n_{L}+7}\left(t_{0}\right)+\left\|\mathbf{u}^{[2]}\left(t, t_{0}\right)\right\|^{2}
\end{aligned}
$$

for all $i=1, \ldots, n_{L}$. Therefore, it is possible to write

$$
\begin{aligned}
& r_{i}^{2}(t)-r_{j}^{2}(t)=z_{3+i}^{2}\left(t_{0}\right)-z_{3+j}^{2}\left(t_{0}\right) \\
& \quad-2\left(\mathbf{s}_{i}-\mathbf{s}_{j}\right) \cdot\left[\mathbf{u}^{[2]}\left(t, t_{0}\right)+\left(t-t_{0}\right) \mathbf{z}_{2}\left(t_{0}\right)\right] \\
& \quad-\left(t-t_{0}\right)^{2}\left(\mathbf{s}_{i}-\mathbf{s}_{j}\right) \cdot \mathbf{z}_{3}\left(t_{0}\right)
\end{aligned}
$$

for all $i, j \in\left\{1, \ldots, n_{L}\right\}$ and $t \in \mathcal{I}$. The comparison between (36) and (38), using (30), gives

$$
\begin{aligned}
& 2\left(t-t_{0}\right)\left(\mathbf{s}_{i}-\mathbf{s}_{j}\right) \cdot\left[\mathbf{x}_{2}\left(t_{0}\right)-\mathbf{z}_{2}\left(t_{0}\right)\right] \\
& \quad+\left(t-t_{0}\right)^{2}\left(\mathbf{s}_{i}-\mathbf{s}_{j}\right) \cdot\left[\mathbf{x}_{3}\left(t_{0}\right)-\mathbf{z}_{3}\left(t_{0}\right)\right]=0
\end{aligned}
$$

for all $i, j \in\left\{1, \ldots, n_{L}\right\}$ and $t \in \mathcal{I}$. Taking the time derivative of both (39) and evaluating at $t=t_{0}$ gives

$$
\left[\begin{array}{c}
\left(\mathbf{s}_{1}-\mathbf{s}_{2}\right)^{T} \\
\left(\mathbf{s}_{1}-\mathbf{s}_{3}\right)^{T} \\
\vdots \\
\left(\mathbf{s}_{n_{L}-2}-\mathbf{s}_{n_{L}}\right)^{T} \\
\left(\mathbf{s}_{n_{L}-1}-\mathbf{s}_{n_{L}}\right)^{T}
\end{array}\right]\left[\mathbf{x}_{2}\left(t_{0}\right)-\mathbf{z}_{2}\left(t_{0}\right)\right]=\mathbf{0}
$$

Again, under Assumption 1 the only solution of (40) is

$$
\mathbf{x}_{2}\left(t_{0}\right)=\mathbf{z}_{2}\left(t_{0}\right)
$$

Taking the second time derivative of (39) and evaluating at $t=t_{0}$ gives

$$
\left[\begin{array}{c}
\left(\mathbf{s}_{1}-\mathbf{s}_{2}\right)^{T} \\
\left(\mathbf{s}_{1}-\mathbf{s}_{3}\right)^{T} \\
\vdots \\
\left(\mathbf{s}_{n_{L}-2}-\mathbf{s}_{n_{L}}\right)^{T} \\
\left(\mathbf{s}_{n_{L}-1}-\mathbf{s}_{n_{L}}\right)^{T}
\end{array}\right]\left[\mathbf{x}_{3}\left(t_{0}\right)-\mathbf{z}_{3}\left(t_{0}\right)\right]=\mathbf{0} .
$$

Again, under Assumption 1 the only solution of (42) is

$$
\mathbf{x}_{3}\left(t_{0}\right)=\mathbf{z}_{3}\left(t_{0}\right)
$$

Finally, comparing (35) with (37) and using (30), (33), (41), and (43) allows us to write

$$
\begin{aligned}
& 2\left(t-t_{0}\right)\left[z_{n_{L}+4}\left(t_{0}\right)-\mathbf{x}_{1}\left(t_{0}\right) \cdot \mathbf{x}_{2}\left(t_{0}\right)\right] \\
& \quad+\left(t-t_{0}\right)^{2}\left(z_{n_{L}+5}\left(t_{0}\right)-\left[\mathbf{x}_{1}\left(t_{0}\right) \cdot \mathbf{x}_{3}\left(t_{0}\right)+\left\|\mathbf{x}_{2}\left(t_{0}\right)\right\|^{2}\right]\right) \\
& \quad+\left(t-t_{0}\right)^{3}\left[z_{n_{L}+6}\left(t_{0}\right)-\mathbf{x}_{2}\left(t_{0}\right) \cdot \mathbf{x}_{3}\left(t_{0}\right)\right] \\
& \quad+\frac{\left(t-t_{0}\right)^{4}}{4}\left[z_{n_{L}+7}\left(t_{0}\right)-\left\|\mathbf{x}_{3}\left(t_{0}\right)\right\|^{2}\right]=0
\end{aligned}
$$


for all $t \in \mathcal{I}$. As the functions $t-t_{0},\left(t-t_{0}\right)^{2},\left(t-t_{0}\right)^{3}$, and $\left(t-t_{0}\right)^{4}$ are linearly independent, it follows that the only solution of (44) is

$$
\left\{\begin{array}{l}
x_{n_{L}+4}\left(t_{0}\right)=\mathbf{x}_{1}\left(t_{0}\right) \cdot \mathbf{x}_{2}\left(t_{0}\right) \\
x_{n_{L}+5}\left(t_{0}\right)=\mathbf{x}_{1}\left(t_{0}\right) \cdot \mathbf{x}_{3}\left(t_{0}\right)+\left\|\mathbf{x}_{2}\left(t_{0}\right)\right\|^{2} \\
x_{n_{L}+6}\left(t_{0}\right)=\mathbf{x}_{2}\left(t_{0}\right) \cdot \mathbf{x}_{3}\left(t_{0}\right) \\
x_{n_{L}+7}\left(t_{0}\right)=\left\|\mathbf{x}_{3}\left(t_{0}\right)\right\|^{2}
\end{array} .\right.
$$

This concludes the proof, as the initial state of (11), which is uniquely determined, matches the initial state of the nonlinear system (8).

Remark 6. The usual concept of observability for nonlinear systems is not as strong as that presented in Theorem 2, see [33] for details. This justifies the need to explicitly detail the observability statement.

Remark 7. The proof of Theorem 2 is specific to the nonlinear system (8) and it follows by relating the original nonlinear system (8) with the nonlinear system (11), regarded as linear time-varying. The nonlinear system (11) has its roots on the original nonlinear system (8) and it was obtained by state augmentation. Hence, by construction, the initial conditions of the original nonlinear system (8) correspond to a subset of the initial conditions of (11). As all initial conditions of (11) are uniquely determined, it follows that the initial conditions of the original nonlinear system (8) are also uniquely determined. This line of reasoning is more general than the proof presented in Theorem 2 and it can be invoked, more generally, when the method of state augmentation is applied as in this paper. Further discussion on this issue can be found in [23].

Remark 8. Before concluding this section, it is important to remark that, although the observability results were derived with respect to the nonlinear system (8), they also apply to the original nonlinear system (6) as they are related through a Lyapunov transformation. Also, the design of an observer for the original nonlinear system follows simply by reversing the state transformation (7) for the corresponding states of the augmented system, as it will be detailed in the following section.

\subsection{Kalman filter}

Although all the results derived so far have been presented in a deterministic setting, in practice there exists measurement noise and often system disturbances. Therefore, a filtering solution is proposed in this section. Theorem 2 provides a constructive result in the sense that a dynamic system with globally exponentially stable error dynamics for the LTV system (8) provides globally exponentially stable error dynamics for the estimation of the state of the nonlinear system. Therefore, a Kalman filter has been designed as follows for the LTV system (11), although other solutions could be devised, e.g., an $\mathcal{H}_{\infty}$ filter.

In order to recover the augmented system dynamics in the original coordinate space, consider the augmented state transformation $\chi(t)=\mathbf{T}_{c}(t) x(t)$, where $\mathbf{T}_{c}(t)=\operatorname{diag}\left(\mathbf{I}, \mathbf{R}^{T}(t)\right.$, $\left.\mathbf{R}^{T}(t), 1, \ldots, 1\right)$. Then, the nominal augmented system dynamics in the original coordinate space are given by

$$
\left\{\begin{array}{l}
\dot{\chi}(t)=\mathcal{A}(t) \chi(t)+\mathbf{B a}(t) \\
\mathbf{y}(t)=\mathbf{C}(t) \chi(t)
\end{array}\right.
$$

where

$\mathcal{A}(t)=\left[\begin{array}{cccccccc}\mathbf{0} & \mathbf{R}(t) & \mathbf{0} & \mathbf{0} & \mathbf{0} & \mathbf{0} & \mathbf{0} & \mathbf{0} \\ \mathbf{0} & -\mathbf{S}(\boldsymbol{\omega}(t)) & \mathbf{I} & \mathbf{0} & \mathbf{0} & \mathbf{0} & \mathbf{0} & \mathbf{0} \\ \mathbf{0} & \mathbf{0} & -\mathbf{S}(\boldsymbol{\omega}(t)) & \mathbf{0} & \mathbf{0} & \mathbf{0} & \mathbf{0} & \mathbf{0} \\ \mathbf{0} & -\frac{\mathbf{s}_{1}^{T} \mathbf{R}(t)}{r_{1}(t)} & \mathbf{0} & 0 & \frac{1}{r_{1}(t)} & 0 & 0 & 0 \\ \vdots & \vdots & \vdots & \vdots & \vdots & \vdots & \vdots & \vdots \\ \mathbf{0} & -\frac{\mathbf{s}_{n_{L}}^{T} \mathbf{R}(t)}{r_{n_{L}}(t)} & \mathbf{0} & 0 & \frac{1}{r_{n_{L}}(t)} & 0 & 0 & 0 \\ \mathbf{a}^{T}(t) \mathbf{R}^{T}(t) & \mathbf{0} & \mathbf{0} & 0 & 0 & 1 & 0 & 0 \\ \mathbf{0} & 2 \mathbf{a}^{T}(t) & \mathbf{0} & 0 & 0 & 0 & 3 & 0 \\ \mathbf{0} & \mathbf{0} & \mathbf{a}^{T}(t) & 0 & 0 & 0 & 0 & 1 \\ \mathbf{0} & \mathbf{0} & \mathbf{0} & 0 & 0 & 0 & 0 & 0\end{array}\right]$.

Including system disturbances and sensor noise to tune the Kalman filter gives the final system dynamics

$$
\left\{\begin{array}{l}
\dot{\chi}(t)=\mathcal{A}(t) \chi(t)+\mathbf{B a}(t)+\mathbf{n}_{x}(t) \\
\mathbf{y}(t)=\mathbf{C}(t) \chi(t)+\mathbf{n}_{y}(t)
\end{array}\right.
$$

where it is assumed that $\mathbf{n}_{x}(t)$ and $\mathbf{n}_{y}(t)$ are uncorrelated, zero-mean, white Gaussian noise processes, with

$$
\mathrm{E}\left[\mathbf{n}_{x}(t) \mathbf{n}_{x}^{T}(\tau)\right]=\mathbf{Q}_{x} \delta(t-\tau)
$$

and

$$
\mathrm{E}\left[\mathbf{n}_{y}(t) \mathbf{n}_{y}^{T}(\tau)\right]=\mathbf{Q}_{y} \delta(t-\tau)
$$

Remark 9. It is important to stress that the filter is not optimal. Indeed, the dependence of the system matrices on the range, acceleration, and angular velocity measurements induce, in the presence of noise on these measurements, multiplicative noise, and the analysis of the stochastic stability of the filter could be carried out. Nevertheless, in a deterministic setting, the filter has globally exponentially 




Fig. 2. Trajectory described by the vehicle.

stable error dynamics and simulation results evidence results comparable with those of the EKF.

\section{SIMULATION RESULTS}

In order to evaluate the performance achieved with the proposed navigation solution, simulations were carried out using a kinematic model for an underwater vehicle. The fact that the full nonlinear dynamics of the vehicle are not considered is not a drawback as the proposed filter relies solely on the vehicle kinematics, which are exact. Therefore, the proposed solution applies to any underwater vehicle, independently of the particular dynamics. The trajectory described by the vehicle is shown in Fig. 2, which moves at $1 \mathrm{~m} / \mathrm{s}$.

Sensor noise was considered for all sensors. In particular, the range, acceleration, and angular velocity measurements are assumed to be corrupted by additive, uncorrelated, zero-mean white Gaussian noise processes, with standard deviations of $1 \mathrm{~m}, 2 \times 10^{-3} \mathrm{~m} / \mathrm{s}^{2}$, and $0.05 \%$, respectively. The attitude, parameterized by roll, pitch, and yaw Euler angles, was also assumed to be corrupted by zero-mean, additive white Gaussian noise, with standard deviation of $0.03^{\circ}$ for the roll and pitch, and $0.3^{\circ}$ for the yaw. The range measurements are assumed to be available at a rate of $1 \mathrm{~Hz}$, while the remaining sensors are sampled at $100 \mathrm{~Hz}$.

The LBL configuration is composed of four acoustic transponders and their positions are

$$
\mathbf{s}_{1}=\left[\begin{array}{c}
0 \\
0 \\
150
\end{array}\right](\mathrm{m}), \mathbf{s}_{2}=\left[\begin{array}{c}
100 \\
0 \\
150
\end{array}\right](\mathrm{m}),
$$

$$
\mathbf{s}_{3}=\left[\begin{array}{c}
0 \\
100 \\
150
\end{array}\right](\mathrm{m}), \mathbf{s}_{4}=\left[\begin{array}{l}
0 \\
0 \\
0
\end{array}\right](\mathrm{m}),
$$

which satisfy Assumption 1 .

The results obtained with the proposed solution are presented in Section 4.1, and its performance compared, in Section 4.2, with that of the extended Kalman filter and a linear position and velocity filter based on the inversion of the range measurements to obtain the position of the vehicle.

Remark 10. In the simulations no range measurement delays were included, for simplicity, but these can be treated in a similar way to [34]. The vehicle displacement during the propagation of the signals was not considered either.

Remark 11. The loss of measurements and outliers is common in acoustic positioning systems. The present framework is beneficial for coping with these as each range measurement can be treated individually. Indeed, when one or more ranges are not available, the filter can either propagate the corresponding states in open-loop (for small periods of time) or simply discard them (for larger periods of time). When a range measurement whose state had been discarded is reacquired, the state of the filter can be augmented again and the corresponding state is initialized with the first measurement, with no impact on the stability of the filter. On the other hand, outliers can be more easily detected at the range level, which is a simple scalar. These are interesting issues for further development with experimental results.

\subsection{Proposed solution}

To tune the Kalman filter, the state disturbance intensity matrix was chosen as $\mathbf{Q}_{x}=10^{-5} \mathbf{I}$ and the output noise intensity matrix as

$$
\mathbf{Q}_{y}=\operatorname{diag}(1,1,1,1,2,2,2,2,2,2) .
$$

The initial conditions were set to zero for the position and velocity. The acceleration of gravity was initialized close to the true value, with $\left[\begin{array}{lll}0 & 0 & 10\end{array}\right]^{T} \mathrm{~m} / \mathrm{s}^{2}$ as the attitude is measured and the magnitude of the acceleration of gravity is usually known. Notice that it would be possible to initialize the position with a close estimate obtained from the inversion of the first set of LBL range measurements. The states corresponding to the range measurements were initialized with the first set of measurements while the remaining states were set to zero, apart from $x_{11}$, which corresponds to the square of the magnitude of the acceleration of gravity, which was initialized with 100 .

The initial evolution of the position, velocity, and acceleration of gravity errors is depicted in Fig. 3, whereas the 

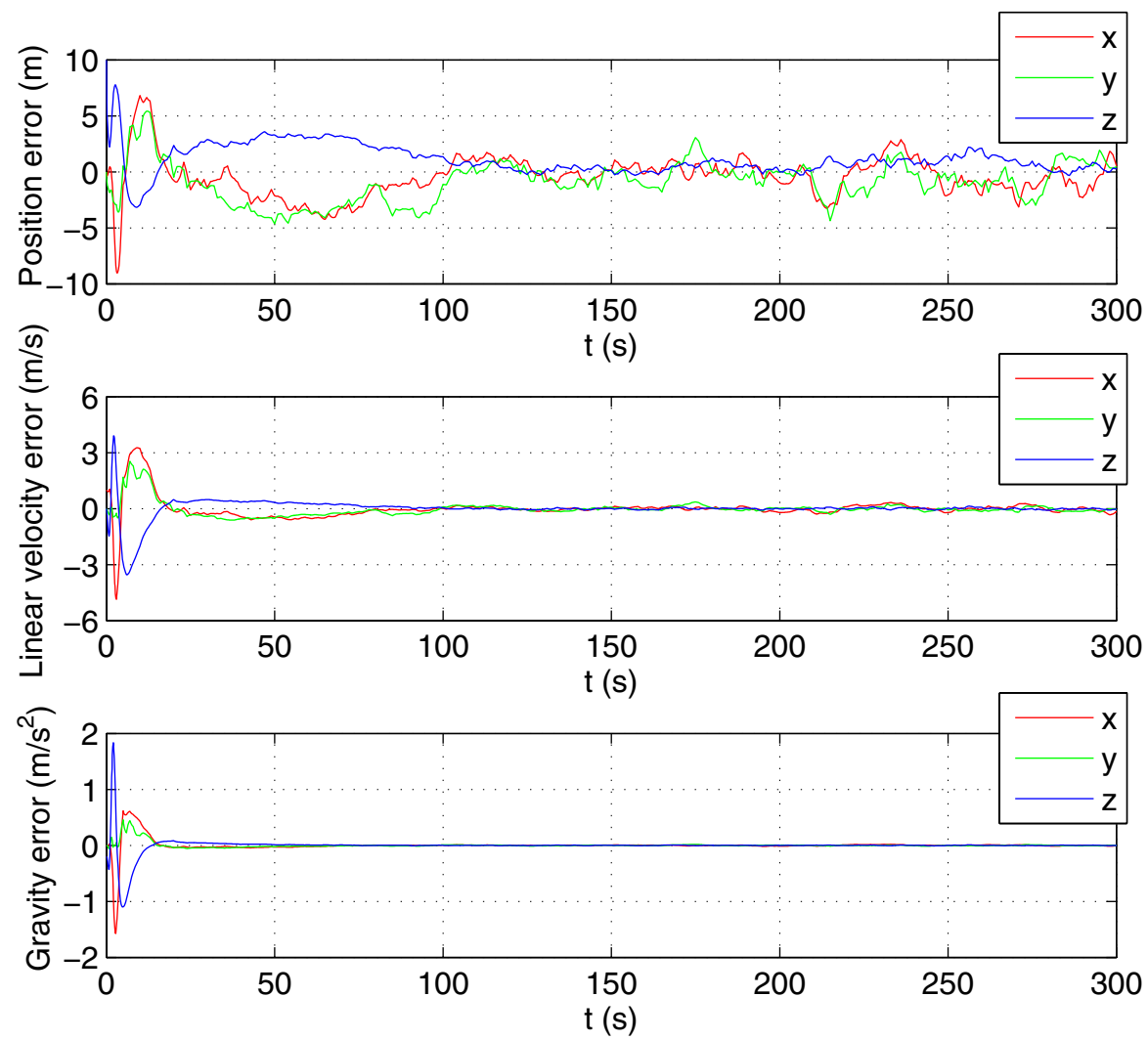

Fig. 3. Initial convergence of the position, velocity, and acceleration of gravity error.

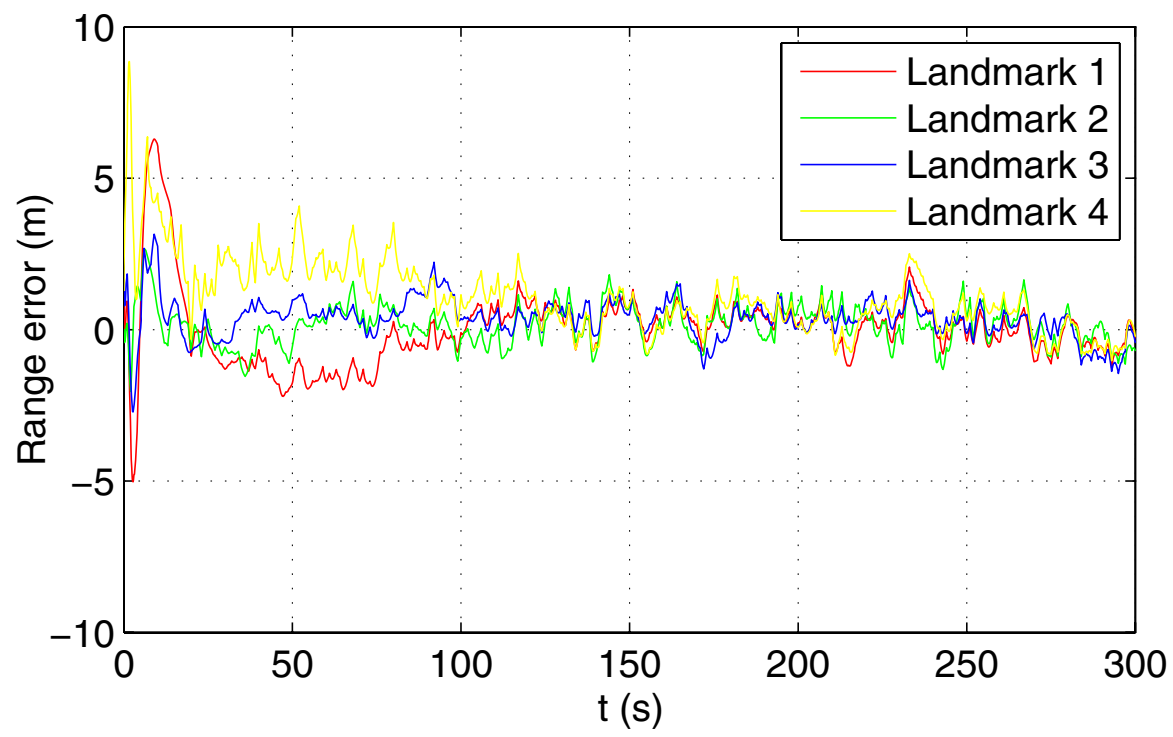

Fig. 4. Initial convergence of the range errors.

initial evolution of the range errors is shown in Fig. 4. The initial convergence of the remaining state errors is shown in Fig. 5. As can be seen from the various plots, the convergence of the filter is quite fast.
In order to better illustrate the performance achieved with the proposed solution, the steady-state errors of the position, velocity, and acceleration of gravity are shown in Fig. 6. Notice that the errors are confined to tight intervals, 


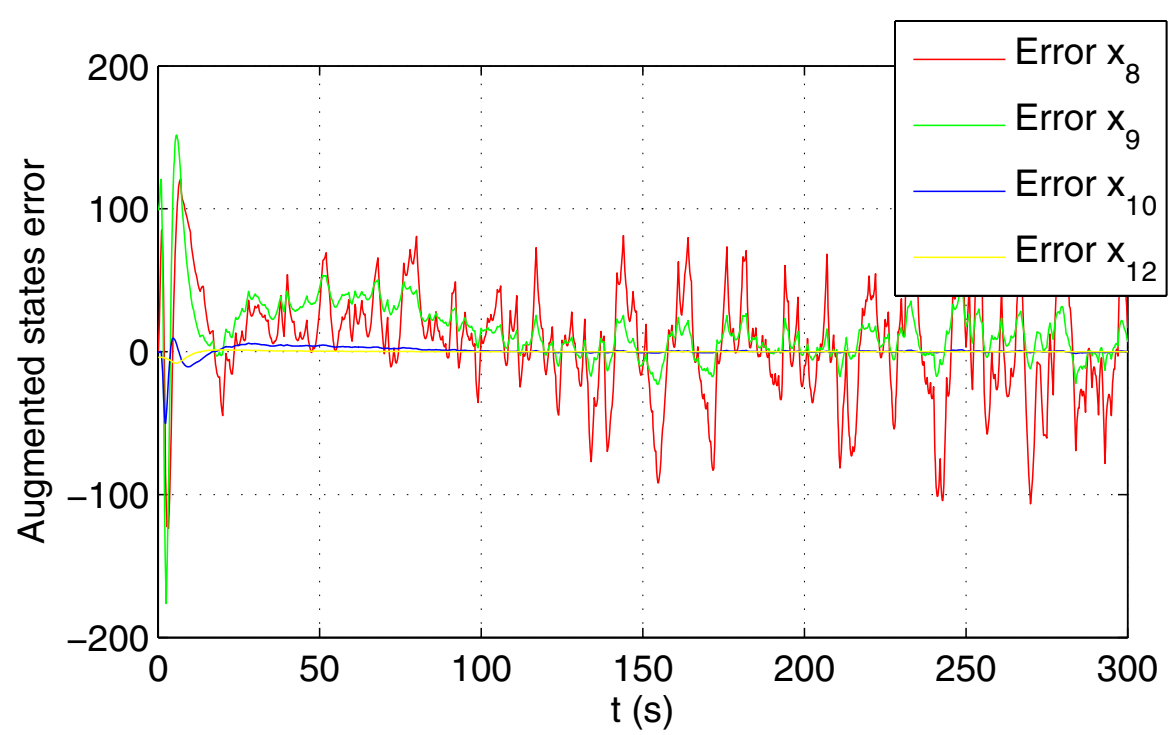

Fig. 5. Initial convergence of the error of the augmented states $x_{8}, x_{9}, x_{10}$, and $x_{11}$.

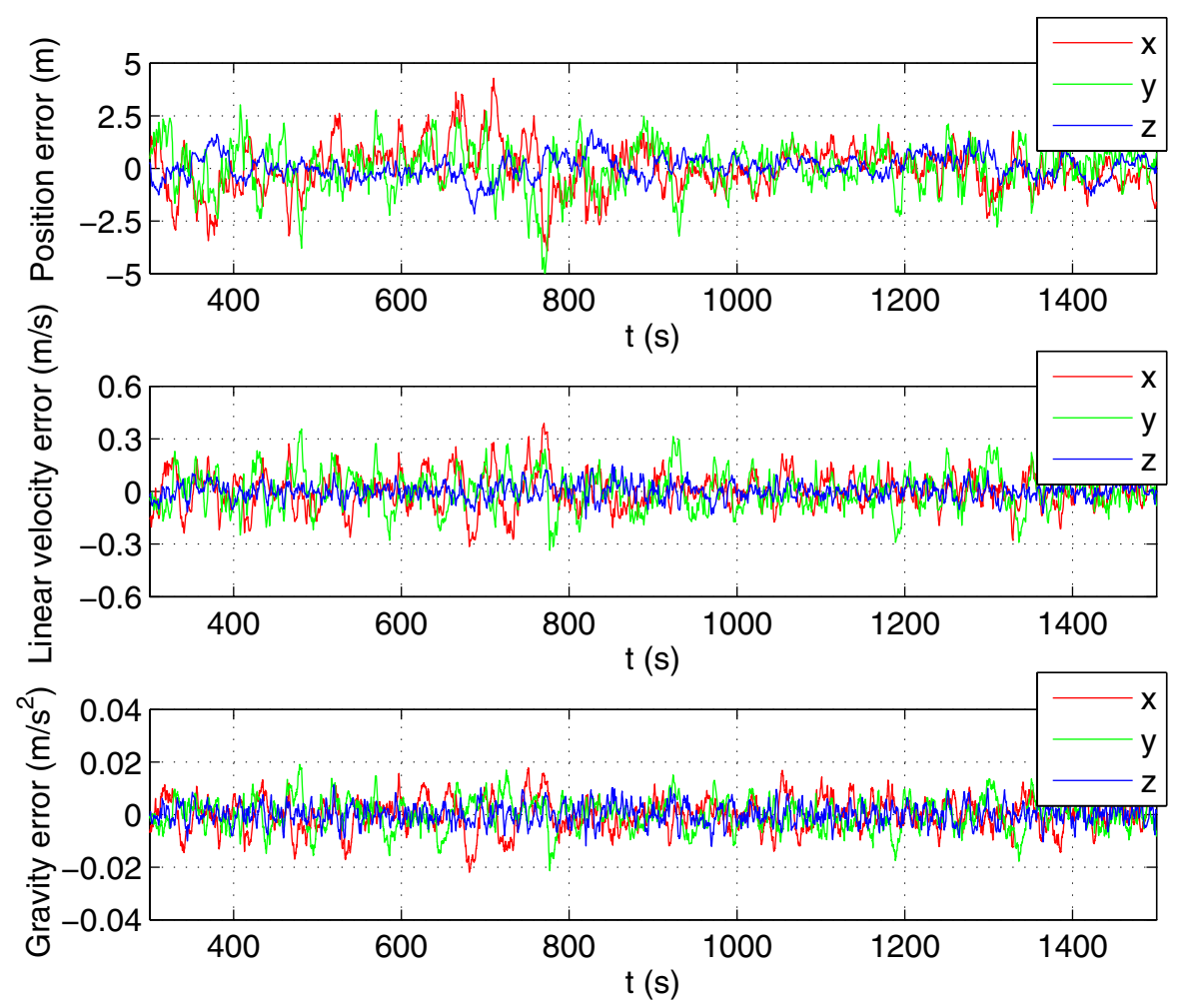

Fig. 6. Detailed evolution of the position, velocity, and acceleration of gravity error.

considering $100 \mathrm{~m}$ baselines, in spite of the realistic measurement noise.

\subsection{Performance comparison}

The proposed filter was compared with two different solutions: the first is the extended Kalman filter applied to the original nonlinear system (6); the second consists in applying the linear Kalman filter proposed in [34] using a position algebraic estimate obtained from the range measurements to feed the filter.

The initial position estimate of the EKF was initialized with an algebraic estimate obtained from the first set of range measurements. The state disturbance intensity matrix was set 


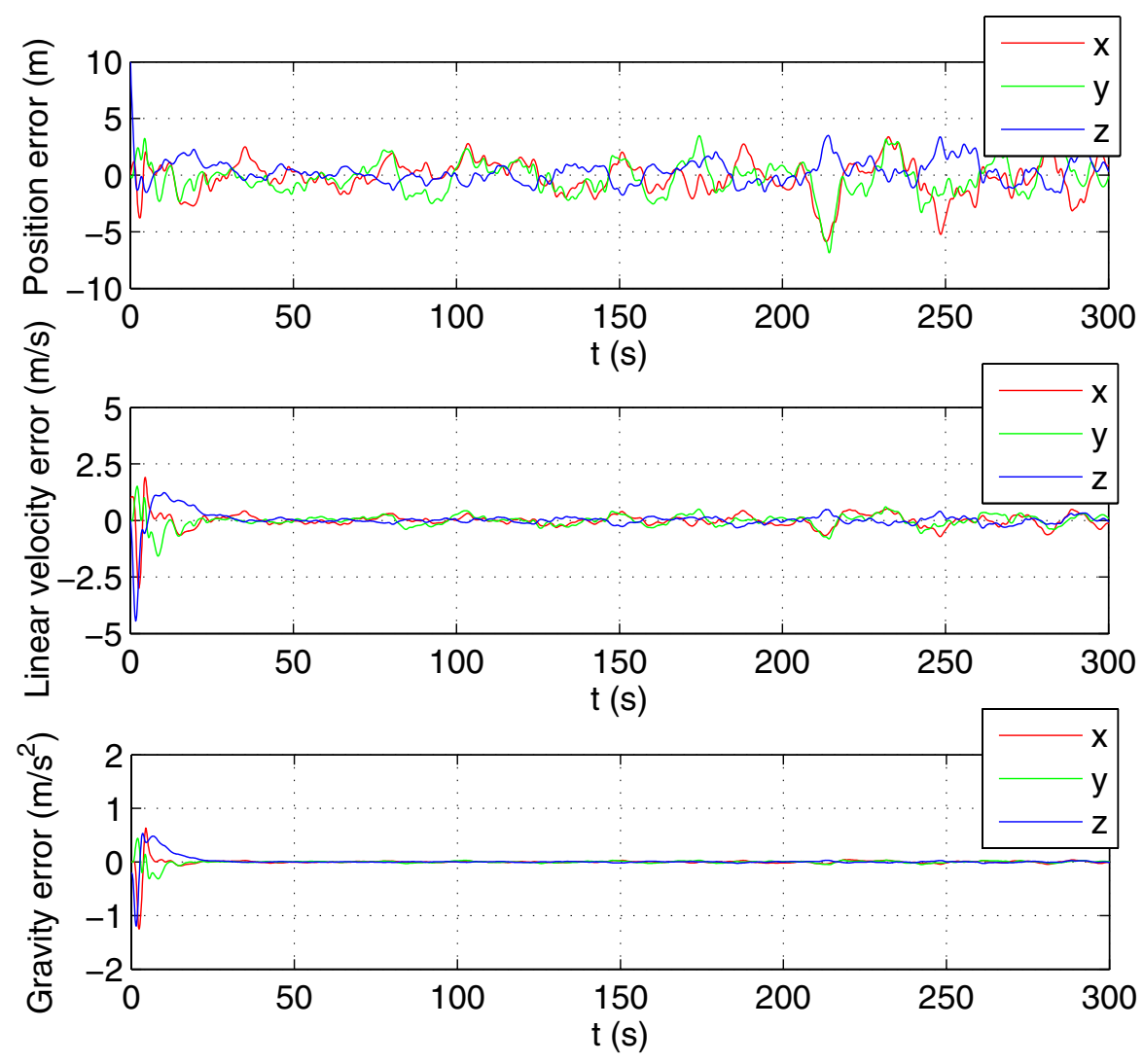

Fig. 7. Initial convergence of the position, velocity, and acceleration of gravity error (linear position filter).

to $\mathbf{Q}_{x}=10^{-5} \mathbf{I}$ and the output noise intensity matrix as $\mathbf{Q}_{y}=\mathbf{I}$, which are equivalent to the ones used in Section 4.1. Although not shown in the paper, the evolution and steady state error of the EKF is very similar to that of the proposed solution. However, the solution proposed in the paper has globally exponentially stable error dynamics, while that has not been shown, to the best of the authors' knowledge, for the EKF.

Finally, simulation results are shown for the linear position and velocity navigation filter proposed in [34] using a position algebraic estimate obtained from the range measurements to feed the filter. The filter parameters are the same as of the EKF, and the initial estimate is also identical. The initial evolution of the filter error is depicted in Fig. 7, whereas the steady-state error is shown in Fig. 8. From the comparison of figures 6 and 8 it is possible to conclude that the performance of the augmented filter is better, particularly for the $z$-axis estimates.

Remark 12. In the simulations, sensor bias was not considered, particularly accelerometer bias. However, it is straightforward to generalize the system dynamics to also include accelerometer bias, while the analysis of observability can be easily inferred from the results presented in [35]. It was chosen not to include that situation in order to focus on the specific subject and contribution of the paper, which is the design of novel sensor-based navigation solutions based on multiple range measurements.

\section{THREE AND TWO RANGE MEASUREMENTS}

So far it has been assumed that at least four noncoplanar landmarks are available, in order to fit the configuration of a long baseline acoustic setup. Although the nonlinear system (8) and the augmented system (11) are general and admit any number of landmarks, the observability depends both on the number and geometric configuration of the landmarks. The specific case of single range measurements is not within the scope of this paper, while the LBL configuration was considered in Section III. The cases that fall between remain. Regardless of the number of landmarks, there exist essentially two additional situations of interest: (i) two landmarks, which give two range measurements; and (ii) three noncolinear landmarks, which give three range measurements. For any other number of landmarks, the observability analysis falls in one of the previous categories: LBL configuration, three 


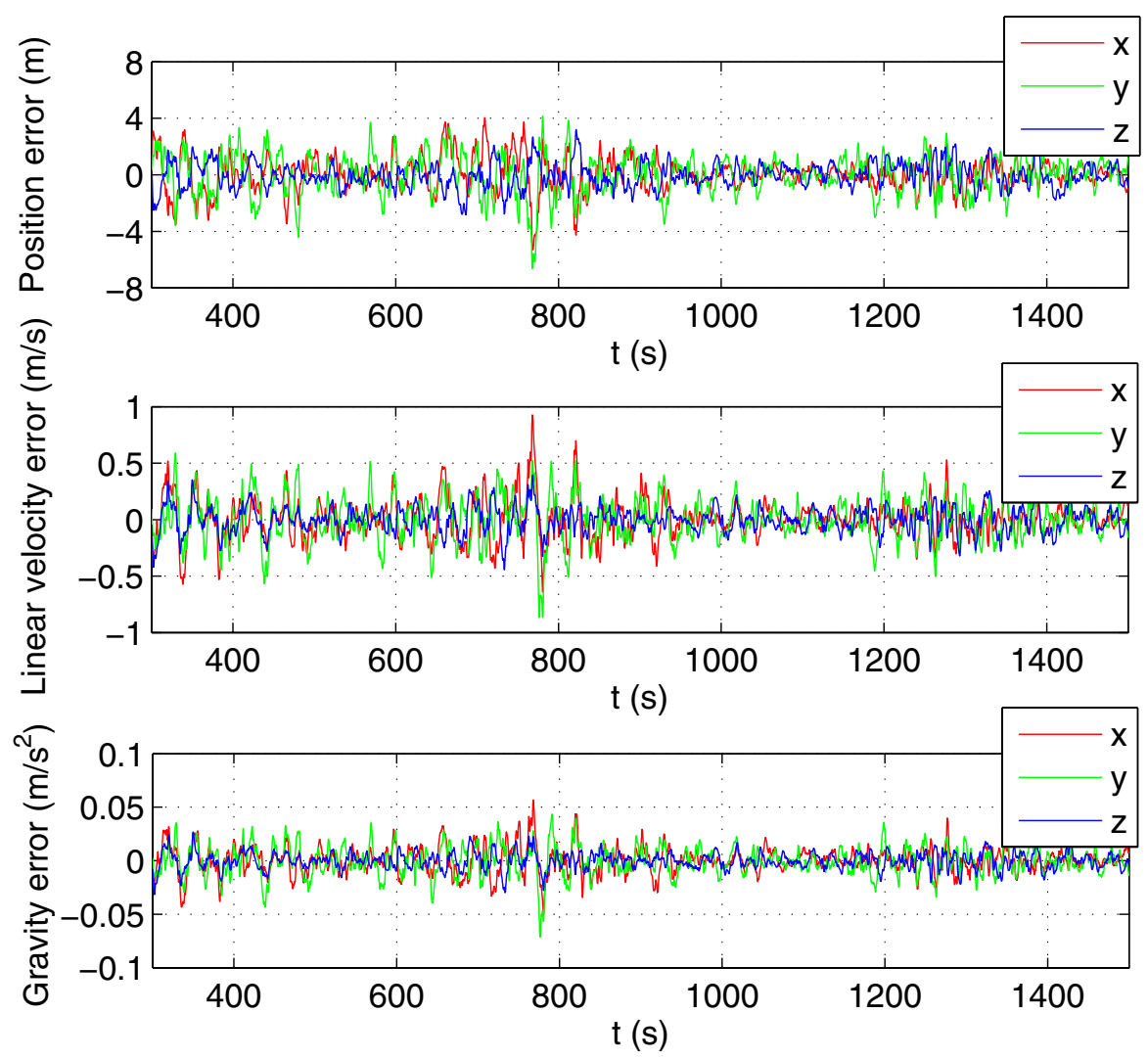

Fig. 8 Detailed evolution of the position, velocity, and acceleration of gravity error (linear position filter).

noncolinear landmarks, two landmarks, or a single landmark. Therefore, in the remainder of this section, only two and three noncolinear landmarks are addressed.

The following theorem addresses the observability of the nonlinear system (11) with three noncolinear landmarks.

Theorem 3. Suppose that Assumption 2 holds and consider the nonlinear system (11), with $n_{L}=3$, and suppose that the three landmarks are noncolinear. Let $\mathbf{d}^{\perp}$ be a unit vector orthogonal to the plane defined by the three landmarks. Then, (11) is observable on $\left[t_{0}, t_{f}\right], t_{0}<t_{f}$, in the sense that any initial condition is uniquely determined by the corresponding response $\left\{\mathbf{y}(t), t \in\left[t_{0}, t_{f}\right]\right\}$ and system input $\left\{\mathbf{u}(t), t \in\left[t_{0}, t_{f}\right]\right\}$, if the set of functions

$$
\begin{aligned}
\mathcal{F}_{3}= & \left\{t-t_{0},\left(t-t_{0}\right)^{2},\left(t-t_{0}\right)^{3},\left(t-t_{0}\right)^{4},\right. \\
& {\left[\mathbf{u}^{[2]}\left(t, t_{0}\right)\right] \cdot \mathbf{d}^{\perp},\left(t-t_{0}\right)\left[\mathbf{u}^{[2]}\left(t, t_{0}\right)\right] \cdot \mathbf{d}^{\perp}, } \\
& \left.\left(t-t_{0}\right)^{2}\left[\mathbf{u}^{[2]}\left(t, t_{0}\right)\right] \cdot \mathbf{d}^{\perp}\right\}
\end{aligned}
$$

is linearly independent on $\left[t_{0}, t_{f}\right]$.

Proof. The proof is essentially a combination of the technical contents of the proof for single range measurements, detailed in [29], and the proof for the LBL configuration. It is presented in Appendix B.
The case of two landmarks is addressed in the following theorem.

Theorem 4. Suppose that Assumption 2 holds and consider the nonlinear system (11), with $n_{L}=2$. Let $\mathbf{d}^{\perp_{1}}$ and $\mathbf{d}^{\perp_{2}}$ denote two unit orthogonal vectors, also orthogonal to the direction defined by the two landmarks. Then, the system (11) is observable on $\left[t_{0}, t_{f}\right], t_{0}<t_{f}$, in the sense that any initial condition is uniquely determined by the corresponding response $\left\{\mathbf{y}(t), t \in\left[t_{0}, t_{f}\right]\right\}$ and system input $\left\{\mathbf{u}(t), t \in\left[t_{0}, t_{f}\right]\right\}$, if the set of functions

$$
\begin{aligned}
\mathcal{F}_{2}= & \left\{t-t_{0},\left(t-t_{0}\right)^{2},\left(t-t_{0}\right)^{3},\left(t-t_{0}\right)^{4},\right. \\
& {\left[\mathbf{u}^{[2]}\left(t, t_{0}\right)\right] \cdot \mathbf{d}^{\perp_{1}},\left(t-t_{0}\right)\left[\mathbf{u}^{[2]}\left(t, t_{0}\right)\right] \cdot \mathbf{d}^{\perp_{1}}, } \\
& \left(t-t_{0}\right)^{2}\left[\mathbf{u}^{[2]}\left(t, t_{0}\right)\right] \cdot \mathbf{d}^{\perp_{1}},\left[\mathbf{u}^{[2]}\left(t, t_{0}\right)\right] \cdot \mathbf{d}^{\perp_{2}}, \\
& \left.\left(t-t_{0}\right)\left[\mathbf{u}^{[2]}\left(t, t_{0}\right)\right] \cdot \mathbf{d}^{\perp_{2}},\left(t-t_{0}\right)^{2}\left[\mathbf{u}^{[2]}\left(t, t_{0}\right)\right] \cdot \mathbf{d}^{\perp_{2}}\right\}
\end{aligned}
$$

is linearly independent on $\left[t_{0}, t_{f}\right]$.

Proof. The proof follows the same steps as that of Theorem 3 and therefore it is omitted.

The conditions stated in Theorems 3 and 4 do not provide, apparently, significant information on the motion constraints that the vehicle must satisfy so that the linear 
motion quantities are observable. Equivalent conditions are established in the following theorem, which allow for appropriate motion planning and control.

Theorem 5. The sets of functions $\mathcal{F}_{3}$ and $\mathcal{F}_{2}$ are linearly independent on $\left[t_{0}, t_{f}\right]$ if and only if the sets of functions

$$
\begin{aligned}
\mathcal{F}_{3}^{*}= & \left\{t-t_{0},\left(t-t_{0}\right)^{2},\left(t-t_{0}\right)^{3},\left(t-t_{0}\right)^{4},\right. \\
& {\left[\mathbf{p}(t)-\mathbf{p}\left(t_{0}\right)\right] \cdot \mathbf{d}^{\perp},\left(t-t_{0}\right)\left[\mathbf{p}(t)-\mathbf{p}\left(t_{0}\right)\right] \cdot \mathbf{d}^{\perp}, } \\
& \left.\left(t-t_{0}\right)^{2}\left[\mathbf{p}(t)-\mathbf{p}\left(t_{0}\right)\right] \cdot \mathbf{d}^{\perp}\right\}
\end{aligned}
$$

and

$$
\begin{aligned}
\mathcal{F}_{2}^{*}= & \left\{t-t_{0},\left(t-t_{0}\right)^{2},\left(t-t_{0}\right)^{3},\left(t-t_{0}\right)^{4},\right. \\
& {\left[\mathbf{p}(t)-\mathbf{p}\left(t_{0}\right)\right] \cdot \mathbf{d}^{\perp_{1}},\left(t-t_{0}\right)\left[\mathbf{p}(t)-\mathbf{p}\left(t_{0}\right)\right] \cdot \mathbf{d}^{\perp_{1}}, } \\
& \left(t-t_{0}\right)^{2}\left[\mathbf{p}(t)-\mathbf{p}\left(t_{0}\right)\right] \cdot \mathbf{d}^{\perp_{1}},\left[\mathbf{p}(t)-\mathbf{p}\left(t_{0}\right)\right] \cdot \mathbf{d}^{\perp_{2}}, \\
& \left(t-t_{0}\right)\left[\mathbf{p}(t)-\mathbf{p}\left(t_{0}\right)\right] \cdot \mathbf{d}^{\perp_{2}}, \\
& \left.\left.\left(t-t_{0}\right)^{2}\left[\mathbf{p}(t)-\mathbf{p}\left(t_{0}\right)\right)\right] \cdot \mathbf{d}^{\perp_{2}}\right\}
\end{aligned}
$$

are linearly independent on $\left[t_{0}, t_{f}\right]$, respectively.

Proof. Substituting $\mathbf{x}_{1}(t)=\mathbf{p}(t)$ in (34) allows to write

$$
\mathbf{u}^{[2]}\left(t, t_{0}\right)=\mathbf{p}(t)-\mathbf{p}\left(t_{0}\right)-\left(t-t_{0}\right) \mathbf{x}_{2}\left(t_{0}\right)-\frac{\left(t-t_{0}\right)^{2}}{2} \mathbf{x}_{3}\left(t_{0}\right) .
$$

The proof follows immediately for the first set of functions by noticing that

$$
\begin{aligned}
& \alpha_{1}\left(t-t_{0}\right)+\alpha_{2}\left(t-t_{0}\right)^{2}+\alpha_{3}\left(t-t_{0}\right)^{3}+\alpha_{4}\left(t-t_{0}\right)^{4} \\
& \quad+\left[\alpha_{5}+\alpha_{6}\left(t-t_{0}\right)\right]\left[\mathbf{u}^{[2]}\left(t, t_{0}\right)\right] \cdot \mathbf{d}^{\perp} \\
& \quad+\alpha_{7}\left(t-t_{0}\right)^{2}\left[\mathbf{u}^{[2]}\left(t, t_{0}\right)\right] \cdot \mathbf{d}^{\perp}=\beta_{1}\left(t-t_{0}\right)+\beta_{2}\left(t-t_{0}\right)^{2} \\
& \quad+\beta_{3}\left(t-t_{0}\right)^{3}+\beta_{4}\left(t-t_{0}\right)^{4}+\beta_{5}\left[\mathbf{p}(t)-\mathbf{p}\left(t_{0}\right)\right] \cdot \mathbf{d}^{\perp} \\
& \quad+\left[\beta_{6}\left(t-t_{0}\right)+\beta_{7}\left(t-t_{0}\right)^{2}\right]\left[\mathbf{p}(t)-\mathbf{p}\left(t_{0}\right)\right] \cdot \mathbf{d}^{\perp}
\end{aligned}
$$

with

$$
\left\{\begin{array}{l}
\beta_{1}=\alpha_{1}-\alpha_{5} \mathbf{x}_{2}\left(t_{0}\right) \cdot \mathbf{d}^{\perp} \\
\beta_{2}=\alpha_{2}-\frac{\alpha_{5}}{2} \mathbf{x}_{3}\left(t_{0}\right) \cdot \mathbf{d}^{\perp}-\alpha_{6} \mathbf{x}_{2}\left(t_{0}\right) \cdot \mathbf{d}^{\perp} \\
\beta_{3}=\alpha_{3}-\frac{\alpha_{6}}{2} \mathbf{x}_{3}\left(t_{0}\right) \cdot \mathbf{d}^{\perp}-\alpha_{7} \mathbf{x}_{2}\left(t_{0}\right) \cdot \mathbf{d}^{\perp} \\
\beta_{4}=\alpha_{4}-\frac{\alpha_{7}}{2} \mathbf{x}_{3}\left(t_{0}\right) \cdot \mathbf{d}^{\perp} \\
\beta_{5}=\alpha_{5} \\
\beta_{6}=\alpha_{6} \\
\beta_{7}=\alpha_{7}
\end{array}\right.
$$

where $\alpha_{i} \in \mathbb{R}, \beta_{i} \in \mathbb{R}, i=1, \ldots, 7$. The proof for the second set of functions is analogous and therefore it is omitted.
Theorems 3 and 4 provide only sufficient conditions on the observability of the range-based nonlinear system with three and two landmarks, respectively. Next, the conservativeness of these conditions is asserted. First, it is shown in the following theorems that certain minimal sets of functions are required to be linearly independent in order for the system to be observable, with three and two landmarks, respectively.

Theorem 6. Consider the nonlinear system (8), with $n_{L}=3$, and suppose that the three landmarks are noncolinear. Let $\mathbf{d}^{\perp}$ be a unit vector orthogonal to the plane defined by the three landmarks. If the set of functions

$$
\begin{aligned}
\mathcal{F}_{3}^{r_{1}}= & \left\{\left[\mathbf{u}^{[2]}\left(t, t_{0}\right)\right] \cdot \mathbf{d}^{\perp},\left(t-t_{0}\right)\left[\mathbf{u}^{[2]}\left(t, t_{0}\right)\right] \cdot \mathbf{d}^{\perp},\right. \\
& \left.\left(t-t_{0}\right)^{2}\left[\mathbf{u}^{[2]}\left(t, t_{0}\right)\right] \cdot \mathbf{d}^{\perp}\right\}
\end{aligned}
$$

is not linearly independent on $\left[t_{0}, t_{f}\right]$, then the system (8) is not observable on $\left[t_{0}, t_{f}\right]$.

Proof. Suppose that the set of functions $\mathcal{F}_{3}^{r_{1}}$ is not linearly independent on $\left[t_{0}, t_{f}\right]$. Then, there exists a unit vector $\left[\begin{array}{ll}\alpha_{1} & \alpha_{2}\end{array}\right.$ $\left.\alpha_{3}\right]^{T}$ such that

$$
\begin{aligned}
& \alpha_{1}\left[\mathbf{u}^{[2]}\left(t, t_{0}\right)\right] \cdot \mathbf{d}^{\perp}+\alpha_{2}\left(t-t_{0}\right)\left[\mathbf{u}^{[2]}\left(t, t_{0}\right)\right] \cdot \mathbf{d}^{\perp} \\
& \quad+\alpha_{3}\left(t-t_{0}\right)^{2}\left[\mathbf{u}^{[2]}\left(t, t_{0}\right)\right] \cdot \mathbf{d}^{\perp}=0
\end{aligned}
$$

for all $t \in\left[t_{0}, t_{f}\right]$. Fix $j \in\{1,2,3\}$ and consider any two initial conditions

$$
\mathbf{x}^{a}\left(t_{0}\right)=\left[\begin{array}{c}
\mathbf{x}_{1}^{a}\left(t_{0}\right) \\
\mathbf{x}_{2}^{a}\left(t_{0}\right) \\
\mathbf{x}_{3}^{a}\left(t_{0}\right)
\end{array}\right]=\left[\begin{array}{c}
\mathbf{s}_{j}+\gamma \alpha_{1} \mathbf{d}^{\perp} \\
\gamma \alpha_{2} \mathbf{d}^{\perp} \\
2 \gamma \alpha_{3} \mathbf{d}^{\perp}
\end{array}\right]
$$

and

$$
\mathbf{x}^{b}\left(t_{0}\right)=\left[\begin{array}{l}
\mathbf{x}_{1}^{b}\left(t_{0}\right) \\
\mathbf{x}_{2}^{b}\left(t_{0}\right) \\
\mathbf{x}_{3}^{b}\left(t_{0}\right)
\end{array}\right]=\left[\begin{array}{c}
\mathbf{s}_{j}-\gamma \alpha_{1} \mathbf{d}^{\perp} \\
-\gamma \alpha_{2} \mathbf{d}^{\perp} \\
-2 \gamma \alpha_{3} \mathbf{d}^{\perp}
\end{array}\right],
$$

where $\gamma \in \mathbb{R} \backslash\{0\}$. Let $r_{i}^{a}(t)$ and $r_{i}^{b}(t), i=1, \ldots, n_{L}$, denote the range readings of the system with initial conditions $\mathbf{x}^{a}\left(t_{0}\right)$ and $\mathbf{x}^{b}\left(t_{0}\right)$, respectively. From (35) it can be seen that

$$
\begin{aligned}
& {\left[r_{i}^{a}(t)\right]^{2}-\left[r_{i}^{b}(t)\right]^{2}=4 \gamma \alpha_{1} \mathbf{d}^{\perp} \cdot \mathbf{u}^{[2]}\left(t, t_{0}\right)} \\
& \quad+4 \gamma\left[\alpha_{2}\left(t-t_{0}\right)+\alpha_{3}\left(t-t_{0}\right)^{2}\right] \mathbf{d}^{\perp} \cdot \mathbf{u}^{[2]}\left(t, t_{0}\right) \\
& \quad+4 \gamma\left[\alpha_{1}+\alpha_{2}\left(t-t_{0}\right)+\alpha_{3}\left(t-t_{0}\right)^{2}\right]\left(\mathbf{s}_{j}-\mathbf{s}_{i}\right) \cdot \mathbf{d}^{\perp}
\end{aligned}
$$

for all $t \in\left[t_{0}, t_{f}\right], i=1,2,3$. For $i \neq j$, notice that, by definition, $\mathbf{d}^{\perp}$ is orthogonal to $\mathbf{s}_{i}-\mathbf{s}_{j}$. This allows us to conclude that $\left(\mathbf{s}_{j}-\mathbf{s}_{i}\right) \cdot \mathbf{d}^{\perp}=0$ for all $i=1,2,3$ and therefore

$$
\begin{aligned}
& {\left[r_{i}^{a}(t)\right]^{2}-\left[r_{i}^{b}(t)\right]^{2}=4 \gamma \alpha_{1} \mathbf{d}^{\perp} \cdot \mathbf{u}^{[2]}\left(t, t_{0}\right)} \\
& \quad+4 \gamma\left[\alpha_{2}\left(t-t_{0}\right)+\alpha_{3}\left(t-t_{0}\right)^{2}\right] \mathbf{d}^{\perp} \cdot \mathbf{u}^{[2]}\left(t, t_{0}\right)
\end{aligned}
$$


for all $t \in\left[t_{0}, t_{f}\right], i=1,2$, 3. Substituting (45) in (46) gives $\left[r_{i}^{a}(t)\right]^{2}-\left[r_{i}^{b}(t)\right]^{2}=0$ for all $t \in\left[t_{0}, t_{f}\right], i=1,2$, 3. As the ranges are positive, this implies that $r_{i}^{a}(t)=r_{i}^{b}(t)$ for all $t \in\left[t_{0}, t_{f}\right], i=1,2,3$, which means that there exist different initial states that yield identical outputs, hence the system is not observable.

Theorem 7. Consider the nonlinear system (8), with $n_{L}=2$. Let $\mathbf{d}^{\perp_{1}}$ and $\mathbf{d}^{\perp_{2}}$ denote two unit orthogonal vectors, also orthogonal to the direction defined by the two landmarks. If the set of functions

$$
\begin{aligned}
& \mathcal{F}_{2}^{r_{1}}=\left\{\left[\mathbf{u}^{[2]}\left(t, t_{0}\right)\right] \cdot \mathbf{d}^{\perp_{1}},\left(t-t_{0}\right)\left[\mathbf{u}^{[2]}\left(t, t_{0}\right)\right] \cdot \mathbf{d}^{\perp_{1}},\right. \\
&\left(t-t_{0}\right)^{2}\left[\mathbf{u}^{[2]}\left(t, t_{0}\right)\right] \cdot \mathbf{d}^{\perp^{1}},\left[\mathbf{u}^{[2]}\left(t, t_{0}\right)\right] \cdot \mathbf{d}^{\perp_{2}}, \\
&\left.\left(t-t_{0}\right)\left[\mathbf{u}^{[2]}\left(t, t_{0}\right)\right] \cdot \mathbf{d}^{\perp_{2}},\left(t-t_{0}\right)^{2}\left[\mathbf{u}^{[2]}\left(t, t_{0}\right)\right] \cdot \mathbf{d}^{\perp_{2}}\right\}
\end{aligned}
$$

is not linearly independent on $\left[t_{0}, t_{f}\right]$, then the system (8) is not observable on $\left[t_{0}, t_{f}\right]$.

Proof. Suppose that the set of functions $\mathcal{F}_{2}^{r_{1}}$ is not linearly independent on $\left[t_{0}, t_{f}\right]$. Then, there exists a unit vector $\left[\alpha_{1} \alpha_{2}\right.$ $\left.\alpha_{3} \alpha_{4} \alpha_{5} \alpha_{6}\right]^{T}$ such that

$$
\begin{aligned}
& {\left[\mathbf{u}^{[2]}\left(t, t_{0}\right)\right] \cdot\left(\alpha_{1} \mathbf{d}^{\perp_{1}}+\alpha_{2} \mathbf{d}^{\perp_{2}}\right)} \\
& \quad+\left(t-t_{0}\right)\left[\mathbf{u}^{[2]}\left(t, t_{0}\right)\right] \cdot\left(\alpha_{3} \mathbf{d}^{\perp_{1}}+\alpha_{4} \mathbf{d}^{\perp_{2}}\right) \\
& \quad+\left(t-t_{0}\right)^{2}\left[\mathbf{u}^{[2]}\left(t, t_{0}\right)\right] \cdot\left(\alpha_{5} \mathbf{d}^{\perp_{1}}+\alpha_{6} \mathbf{d}^{\perp_{2}}\right)=0
\end{aligned}
$$

for all $t \in\left[t_{0}, t_{f}\right]$. Fix $j \in\{1,2\}$ and consider any two initial conditions

$$
\mathbf{x}^{a}\left(t_{0}\right)=\left[\begin{array}{c}
\mathbf{x}_{1}^{a}\left(t_{0}\right) \\
\mathbf{x}_{2}^{a}\left(t_{0}\right) \\
\mathbf{x}_{3}^{a}\left(t_{0}\right)
\end{array}\right]=\left[\begin{array}{c}
\mathbf{s}_{j}+\gamma \alpha_{1} \mathbf{d}^{\perp_{1}}+\gamma \alpha_{2} \mathbf{d}^{\perp_{2}} \\
\gamma \alpha_{3} \mathbf{d}^{\perp_{1}}+\gamma \alpha_{4} \mathbf{d}^{\perp_{2}} \\
\gamma \alpha_{5} \mathbf{d}^{\perp_{1}}+\gamma \alpha_{6} \mathbf{d}^{\perp_{2}}
\end{array}\right]
$$

and

$$
\mathbf{x}^{b}\left(t_{0}\right)=\left[\begin{array}{l}
\mathbf{x}_{1}^{b}\left(t_{0}\right) \\
\mathbf{x}_{2}^{b}\left(t_{0}\right) \\
\mathbf{x}_{3}^{b}\left(t_{0}\right)
\end{array}\right]=\left[\begin{array}{c}
\mathbf{s}_{j}-\gamma \alpha_{1} \mathbf{d}^{\perp_{1}}-\gamma \alpha_{2} \mathbf{d}^{\perp_{2}} \\
-\gamma \alpha_{3} \mathbf{d}^{\perp_{1}}-\gamma \alpha_{4} \mathbf{d}^{\perp_{2}} \\
-\gamma \alpha_{5} \mathbf{d}^{\perp_{1}}-\gamma \alpha_{6} \mathbf{d}^{\perp_{2}}
\end{array}\right],
$$

where $\gamma \in \mathbb{R} \backslash\{0\}$. The remainder of the proof amounts to show that the outputs of the system for these two different initial conditions are identical. This follows the same steps of Theorem 6 and therefore it is omitted.

Comparing the set of functions $F_{3}$ and $\mathcal{F}_{3}^{r_{1}}$, or $F_{2}$ and $\mathcal{F}_{2}^{r_{1}}$, it seems that there is some conservativeness by including the polynomials $t-t_{0},\left(t-t_{0}\right)^{2},\left(t-t_{0}\right)^{3}$, and $\left(t-t_{0}\right)^{4}$. The following theorems show that these polynomials influence the observability of the system.

Theorem 8. Consider the nonlinear system (8), with $n_{L}=3$, and suppose that the 3 landmarks are noncolinear. Let $\mathbf{d}^{\perp}$ be a unit vector orthogonal to the plane defined by the three landmarks. Suppose that there exists a non-null vector $\left[\alpha_{1} \alpha_{2}\right]^{T}$ such that

$$
\left[\mathbf{u}^{[2]}\left(t, t_{0}\right)\right] \cdot \mathbf{d}^{\perp}=\alpha_{1}\left(t-t_{0}\right)+\alpha_{2}\left(t-t_{0}\right)^{2}
$$

for all $t \in\left[t_{0}, t_{f}\right]$. Then the system (8) is not observable on $\left[t_{0}, t_{f}\right]$.

Proof. Fix $j \in\{1,2,3\}$ and consider any two initial conditions

$$
\mathbf{x}^{a}\left(t_{0}\right)=\left[\begin{array}{c}
\mathbf{x}_{1}^{a}\left(t_{0}\right) \\
\mathbf{x}_{2}^{a}\left(t_{0}\right) \\
\mathbf{x}_{3}^{a}\left(t_{0}\right)
\end{array}\right]=\left[\begin{array}{c}
\mathbf{s}_{j}+\gamma \mathbf{d}^{\perp} \\
\mathbf{0} \\
\mathbf{0}
\end{array}\right]
$$

and

$$
\mathbf{x}^{b}\left(t_{0}\right)=\left[\begin{array}{l}
\mathbf{x}_{1}^{b}\left(t_{0}\right) \\
\mathbf{x}_{2}^{b}\left(t_{0}\right) \\
\mathbf{x}_{3}^{b}\left(t_{0}\right)
\end{array}\right]=\left[\begin{array}{c}
\mathbf{s}_{j}-\gamma \mathbf{d}^{\perp} \\
-2 \alpha_{1} \mathbf{d}^{\perp} \\
-4 \alpha_{2} \mathbf{d}^{\perp}
\end{array}\right],
$$

where $\gamma \in \mathbb{R}$. Let $r_{i}^{a}(t)$ and $r_{i}^{b}(t), i=1, \ldots, n_{L}$ denote the range readings of the system with initial conditions $\mathbf{x}^{a}\left(t_{0}\right)$ and $\mathbf{x}^{b}\left(t_{0}\right)$, respectively. From (35) it is straightforward to show that

$$
\begin{aligned}
& {\left[r_{i}^{a}(t)\right]^{2}-\left[r_{i}^{b}(t)\right]^{2}=-4\left(t-t_{0}\right) \gamma \alpha_{1}-4\left(t-t_{0}\right)^{2} \gamma \alpha_{2}} \\
& \quad-4\left(t-t_{0}\right)^{2}\left[\alpha_{1}+\alpha_{2}\left(t-t_{0}\right)\right]^{2} \\
& \quad+4\left[\gamma+\alpha_{1}\left(t-t_{0}\right)+\alpha_{2}\left(t-t_{0}\right)^{2}\right] \mathbf{d}^{\perp} \cdot \mathbf{u}^{[2]}\left(t, t_{0}\right) \\
& \quad+4\left(\gamma+\alpha_{1}+\alpha_{2}\right)\left(\mathbf{s}_{j}-\mathbf{s}_{i}\right) \cdot \mathbf{d}^{\perp}
\end{aligned}
$$

for all $t \in\left[t_{0}, t_{f}\right], i=1,2,3$. For $i \neq j$, notice that, by definition, $\mathbf{d}^{\perp}$ is orthogonal to $\mathbf{s}_{i}-\mathbf{s}_{j}$. This allows to conclude that $\left(\mathbf{s}_{j}-\mathbf{s}_{i}\right) \cdot \mathbf{d}^{\perp}=\mathbf{0}$ for all $i=1,2,3$ and therefore

$$
\begin{aligned}
& {\left[r_{i}^{a}(t)\right]^{2}-\left[r_{i}^{b}(t)\right]^{2}=-4\left(t-t_{0}\right) \gamma \alpha_{1}} \\
& \quad-4\left(t-t_{0}\right)^{2} \gamma \alpha_{2}-4\left(t-t_{0}\right)^{2}\left[\alpha_{1}+\alpha_{2}\left(t-t_{0}\right)\right]^{2} \\
& \quad+4\left[\gamma+\alpha_{1}\left(t-t_{0}\right)+\alpha_{2}\left(t-t_{0}\right)^{2}\right] \mathbf{d}^{\perp} \cdot \mathbf{u}^{[2]}\left(t, t_{0}\right)
\end{aligned}
$$

for all $t \in\left[t_{0}, t_{f}\right], i=1,2,3$. Substituting (47) in (48) gives $\left[r_{i}^{a}(t)\right]^{2}-\left[r_{i}^{b}(t)\right]^{2}=0$ for all $t \in\left[t_{0}, t_{f}\right], i=1,2,3$. As the ranges are positive, this implies that $r_{i}^{a}(t)=r_{i}^{b}(t)$ for all $t \in\left[t_{0}, t_{f}\right], i=1,2,3$, which means that there exist different initial states that yield the identical outputs, hence the system is not observable.

Theorem 9. Consider the nonlinear system (8), with $n_{L}=2$. Let $\mathbf{d}^{\perp_{1}}$ and $\mathbf{d}^{\perp_{2}}$ denote two unit orthogonal vectors, also orthogonal to the direction defined by the two landmarks and suppose that there exists a non-null vector $\left[\begin{array}{ll}\alpha_{1} & \alpha_{2}\end{array}\right]^{T}$ such that

$$
\left[\mathbf{u}^{[2]}\left(t, t_{0}\right)\right] \cdot \mathbf{d}^{\perp_{1}}=\alpha_{1}\left(t-t_{0}\right)+\alpha_{2}\left(t-t_{0}\right)^{2}
$$

or 


$$
\left[\mathbf{u}^{[2]}\left(t, t_{0}\right)\right] \cdot \mathbf{d}^{\perp_{2}}=\alpha_{1}\left(t-t_{0}\right)+\alpha_{2}\left(t-t_{0}\right)^{2}
$$

for all $t \in\left[t_{0}, t_{f}\right]$. Then the system (8) is not observable on $\left[t_{0}, t_{f}\right]$.

Proof. The proof is similar to the proof of Theorem 8 and therefore it is omitted.

\section{CONCLUSIONS}

This paper presented a novel sensor-based long baseline position and velocity navigation filter for underwater vehicles based directly on the sensor measurements. Traditional solutions resort either to the extended Kalman filter or to solutions based on position algebraic estimates obtained from the range measurements. The solution presented herein departs from previous approaches as the range measurements are explicitly embedded in the filter design, therefore avoiding inversion algorithms. Moreover, the nonlinear system dynamics are considered to their full extent and no linearizations are carried out whatsoever.

State augmentation is at the core of the proposed framework. Indeed, the proposed system has $13+n_{L}$ states, which compares to a minimum of nine states for traditional solutions. This system can be regarded as LTV which allows the implementation of a GES Kalman filter to estimate the system state. The achieved performance coupled with the guarantee of global asymptotic stability justifies the additional computational complexity. In addition to that, the framework allows for any number of range measurements, and only the observability of the system varies, as expected. The specific cases of two and three range measurements, which fall into a framework between single range measurements and the LBL configuration, were also analyzed in detail. Additional sensors, like depth sensors, are also trivially added to the filtering framework, without any significant additional computational complexity. An alternative framework, which employs relative velocity readings instead of acceleration readings, is also easily devised, following steps similar to those detailed in [28] but considering the LBL structure presented in this paper.

The performance of the proposed solution was evaluated in a simulation environment. It was shown that the filter achieves similar performance to the extended Kalman filter and outperforms linear position and velocity filters based on position algebraic estimates obtained directly from the range measurements. Finally, it is important to stress that, although the solution derived in the paper was applied for AUVs, it is general and easily applied to any autonomous vehicle equipped with an LBL range system.

\section{REFERENCES}

1. Sukkarieh, S., E. Nebot, and H. Durrant-Whyte, "A high integrity IMU/GPS navigation loop for autonomous land vehicle applications," IEEE Trans. Robot. Autom., Vol. 15, No. 3, pp. 572-578 (1999).

2. Yun, X., E. Bachmann, R. McGhee, R. Whalen, R. Roberts, R. Knapp, A. Healey, and M. Zyda, "Testing and evaluation of an integrated GPS/INS system for small AUV navigation," IEEE J. Ocean. Eng., Vol. 24, No. 3, pp. 396-404 (1999).

3. Hajiyev, C., "GNSS Signals Processing via Linear and Extended Kalman Filters," Asian J. Control, Vol. 13, No. 2, pp. 273-282 (2011).

4. Yeh, F.-K., H.-W. Chen, and Y.-H. Tsai, "Using Delta Range for Fault Detection/Exclusion on GPS Positioning," Asian J. Control, Vol. 14, No. 4, pp. 936-946 (2012).

5. Vik, B. and T. Fossen, "A nonlinear observer for GPS and INS integration," In Proc. of the 40th IEEE Conf. on Decision and Control, Vol. 3, pp. 2956-2961, Orlando, FL, USA (December 2001).

6. Jouffroy, J. and J. Opderbecke, "Underwater vehicle trajectory estimation using contracting PDE-based observers," In Proc. of the 2004 American Control Conf. Vol. 5, pp. 4108-4113, Boston, MA, USA (Jun-July 2004).

7. Kinsey, J. and L. Whitcomb, "Preliminary field experience with the DVLNAV integrated navigation system for manned and unmanned submersibles," In Proc. of the 1st IFAC workshop on Guidance and Control of Underwater Vehicles, pp. 83-88, Newport, South Wales, UK (April 2003).

8. Larsen, M., "Synthetic long baseline navigation of underwater vehicles," In Proc. of the 2000 MTS/IEEE Oceans, Vol. 3, pp. 2043-2050, Providence, RI, USA (September 2000).

9. Vaganay, J., J. Bellingham, and J. Leonard, "Comparison of fix computation and filtering for autonomous acoustic navigation," Int. J. Syst. Sci., Vol. 29, No. 10, pp. 11111122 (1998).

10. Youngberg, J., Method for extending GPS to underwater applications (June 1992). US Patent 5119341

11. Thomas, H., "GIB buoys: an interface between space and depths of the oceans," In Proc. of the 1998 Workshop on Autonomous Underwater Vehicles, pp. 181-184, Cambridge, MA, USA (August 1998).

12. Alcocer, A., P. Oliveira, and A. Pascoal, "Study and Implementation of an EKF GIB-based Underwater Positioning System," Control Eng. Practice, Vol. 15, No. 6, pp. 689-701 (2007).

13. Batista, P., C. Silvestre, and P. Oliveira, "Optimal position and velocity navigation filters for autonomous vehicles," Automatica, Vol. 46, No. 4, pp. 767-774 (2010). 
14. Morgado, M., P. Oliveira, C. Silvestre, and J. Vasconcelos, "USBL/INS Tightly-Coupled Integration Technique for Underwater Vehicles," In Proc. of the 9th Int. Conf. on Information Fusion, Florence, Italy (July 2006).

15. Newman, P. and J. Leonard, "Pure Range-Only Sub-Sea SLAM," In Proc. of the 2003 IEEE Conf. on Robotics and Automation, Vol. 2, pp. 1921-1926, Taipei, Taiwan (September 2003).

16. Ross, A. and J. Jouffroy, "Remarks on the observability of single beacon underwater navigation," In Proc. of the 14th International Symposium on Unmanned Untethered Submersible Technology, Durham, NH, USA (August 2005).

17. Blanco, J.-L., J. Gonzalez, and J.-A. Fernandez-Madrigal, "A Pure Probabilistic Approach to Range-Only SLAM," In Proc. of the 2008 IEEE Conf. on Robotics and Automation, pp. 1436-1441, Pasadena, CA, USA (May 2008).

18. Olson, E., J. Leonard, and S. Teller, "Robust Range-Only Beacon Localization," IEEE J. Ocean. Eng., Vol. 31, No. 4, pp. 949-958 (2006).

19. Gadre, A. and D. Stilwell, "A complete solution to underwater navigation in the presence of unknown currents based on range measurements from a single location," In Proc. of the 2005 IEEE/RSJ Int. Conf. on Intelligent Robots and Systems, pp. 1420-1425, Edmonton, AB, Canada (August 2005).

20. Casey, T., B. Guimond, and J. Hu, "Underwater Vehicle Positioning Based on Time of Arrival Measurements from a Single Beacon," In Proc. of the MTS/IEEE Oceans 2007, pp. 1-8, Vancouver, BC, Canada (Sept.Oct. 2007).

21. Jouffroy, J. and J. Reger, "An algebraic perspective to single-transponder underwater navigation," In Proc. of the 2006 IEEE Int. Conf. on Control Applications, pp. 1789-1794, Munich, Germany (October 2006).

22. Antonelli, G., F. Arrichiello, S. Chiaverini, and G. Sukhatme, "Observability analysis of relative localization for AUVs based on ranging and depth measurements," In Proc. of the 2010 IEEE Int. Conf. on Robotics and Automation, pp. 4286-4271, Anchorage, AK, USA (May 2010).

23. Parlangeli, G., T. Pedone, and G. Indiveri, "Relative Pose Observability Analysis for 3D Nonholonomic Vehicles Based on Range Measurements Only," In Proc. of the 9th IFAC Conference on Manoeuvring and Control of Marine Craft, Arenzano, Italy (September 2012).
24. Webster, S., R. Eustice, H. Singh, and L. Whitcomb, "Preliminary deep water results in single-beacon oneway-travel-time acoustic navigation for underwater vehicles," In Proc. of the 2009 IEEE/RSJ Int. Conf. on Intelligent Robots and Systems-IROS 2009, pp. 20532060, Saint Louis, MO, USA (October 2009).

25. Kinsey, J., R. Eustice, and L. Whitcomb, "A Survey of Underwater Vehicle Navigation: Recent Advances and New Challenges," In Proc. of the 7th IFAC Conf. on Manoeuvring and Control of Marine Craft, Lisboa, Portugal (September 2006).

26. Leonard, J., A. Bennett, C. Smith, and H. Feder, Autonomous underwater vehicle navigation. Technical Report Technical Memorandum 98-1, MIT Marine Robotics Laboratory (1998).

27. Batista, P., C. Silvestre, and P. Oliveira, "A Sensor-based Long Baseline Position and Velocity Navigation Filter for Underwater Vehicles," In Proc. of the 8th IFAC Symposium on Nonlinear Control Systems-NOLCOS 2010, pp. 302-307, Bologna, Italy (September 2010).

28. Batista, P., C. Silvestre, and P. Oliveira, "Single Range Navigation in the presence of Constant Unknown Drifts," In Proc. of the 2009 European Control Conf., pp. 39833988, Budapest, Hungary (August 2009).

29. Batista, P., C. Silvestre, and P. Oliveira, "Single Beacon Navigation: Observability Analysis and Filter Design," In Proc. of the 2010 American Control Conf., pp. 61916196, Baltimore, MD, USA (Jun.-Jul. 2010).

30. Batista, P., C. Silvestre, and P. Oliveira, "Single Range Aided Navigation and Source Localization: observability and filter design," Syst. Control Lett., Vol. 60, No. 8, pp. 665-673 (2011).

31. Morgado, M., P. Batista, P. Oliveira, and C. Silvestre, "Position USBL/DVLSensor-based Navigation Filter in the presence of Unknown Ocean Currents," Automatica, Vol. 47, No. 12, pp. 2604-2614 (2011).

32. Kelly, A., Modern Inertial and Satellite Navigation Systems. Technical Report CMU-RI-TR-94-15, Robotics Institute, Carnegie Mellon University, Pittsburgh, PA, USA (May 1994).

33. Hermann, R. and A. Krener, "Nonlinear controllability and observability," IEEE Trans. Autom. Control, Vol. 22, No. 5, pp. $728-740$ (October 1977).

34. Batista, P., C. Silvestre, and P. Oliveira, "Position and Velocity Optimal Sensor-based Navigation Filters for UAVs," In Proc. of the 2009 American Control Conf., pp. 5404-5409, Saint Louis, MO, USA (June 2009).

35. Batista, P., C. Silvestre, and P. Oliveira, "On the observability of linear motion quantities in navigation systems," Syst. Control Lett., Vol. 60, No. 2, pp. 101-110 (2011). 


\section{APPENDIX A}

\subsection{TRANSITION MATRIX}

The transition matrix $\phi\left(t, t_{0}\right)$ associated with the system matrix $\mathbf{A}(t)$ given by (9) can be computed resorting to the Peano-Baker series, which in this case results in the sum of the first five terms only, as the remaining are null.

Straightforward but long and tedious computations allow us to write

$$
\phi\left(t, t_{0}\right)=\left[\begin{array}{ccc}
\phi_{A A}\left(t, t_{0}\right) & \mathbf{0} & \mathbf{0} \\
\phi_{B A}\left(t, t_{0}\right) & \mathbf{I} & \phi_{B C}\left(t, t_{0}\right) \\
\phi_{C A}\left(t, t_{0}\right) & \mathbf{0} & \phi_{C C}\left(t, t_{0}\right)
\end{array}\right],
$$

where

$$
\begin{aligned}
& \phi_{A A}\left(t, t_{0}\right)=\left[\begin{array}{ccc}
\mathbf{I} & \left(t-t_{0}\right) \mathbf{I} & \frac{\left(t-t_{0}\right)^{2}}{2} \mathbf{I} \\
\mathbf{0} & \mathbf{I} & \left(t-t_{0}\right) \mathbf{I} \\
\mathbf{0} & \mathbf{0} & \mathbf{I}
\end{array}\right], \\
& \phi_{B A}\left(t, t_{0}\right)=\left[\phi_{B A 1}\left(t, t_{0}\right) \quad \phi_{B A 2}\left(t, t_{0}\right) \quad \phi_{B A 3}\left(t, t_{0}\right)\right], \\
& \phi_{B A 1}\left(t, t_{0}\right)=\left[\begin{array}{c}
\int_{t_{0}}^{t} \frac{\left[\mathbf{u}^{[1]}\left(\sigma, t_{0}\right)\right]^{T}}{r_{1}(\sigma)} d \sigma \\
\vdots \\
\int_{t_{0}}^{t} \frac{\left[\mathbf{u}^{[1]}\left(\sigma, t_{0}\right)\right]^{T}}{r_{n_{L}}(\sigma)} d \sigma
\end{array}\right],
\end{aligned}
$$

$$
\begin{aligned}
& \phi_{B A 2}\left(t, t_{0}\right) \\
& =\left[\begin{array}{c}
\int_{t_{0}}^{t} \frac{-\mathbf{s}_{1}^{T}+\left(\sigma-t_{0}\right)\left[\mathbf{u}^{[1]}\left(\sigma, t_{0}\right)\right]^{T}+\left[\mathbf{u}^{[2]}\left(\sigma, t_{0}\right)\right]^{T}}{r_{1}(\sigma)} d \sigma \\
\vdots \\
\int_{t_{0}}^{t} \frac{-\mathbf{s}_{n_{L}}^{T}+\left(\sigma-t_{0}\right)\left[\mathbf{u}^{[1]}\left(\sigma, t_{0}\right)\right]^{T}+\left[\mathbf{u}^{[2]}\left(\sigma, t_{0}\right)\right]^{T}}{r_{n_{L}}(\sigma)} d \sigma
\end{array}\right], \\
& \phi_{B A 3}\left(t, t_{0}\right)=\left[\begin{array}{c}
\phi_{B A 3,1}\left(t, t_{0}\right) \\
\phi_{B A 3,2}\left(t, t_{0}\right) \\
\vdots \\
\phi_{B A 3, n_{L}}\left(t, t_{0}\right)
\end{array}\right], \\
& \phi_{B A 3, i}\left(t, t_{0}\right)=-\int_{t_{0}}^{t} \frac{\left(\sigma-t_{0}\right) \mathbf{s}_{i}^{T}}{r_{i}(\sigma)} d \sigma \\
& +\int_{t_{0}}^{t} \frac{\frac{\left(\sigma-t_{0}\right)^{2}}{2}\left[\mathbf{u}^{[1]}\left(\sigma, t_{0}\right)\right]^{T}}{r_{i}(\sigma)} d \sigma \\
& +\int_{t_{0}}^{t} \frac{\left(\sigma-t_{0}\right)\left[\mathbf{u}^{[2]}\left(\sigma, t_{0}\right)\right]^{T}}{r_{i}(\sigma)} d \sigma, i=1, \ldots, n_{L},
\end{aligned}
$$

$$
\phi_{B C}\left(t, t_{0}\right)=\left[\begin{array}{ccc}
\int_{t_{0}}^{t} \frac{1}{r_{1}(\sigma)} d \sigma & \ldots & \int_{t_{0}}^{t} \frac{1}{r_{n_{L}}(\sigma)} d \sigma \\
\int_{t_{0}}^{t} \frac{\sigma-t_{0}}{r_{1}(\sigma)} d \sigma & \cdots & \int_{t_{0}}^{t} \frac{\sigma-t_{0}}{r_{n_{L}}(\sigma)} d \sigma \\
\int_{t_{0}}^{t} \frac{3}{2} \frac{\left(\sigma-t_{0}\right)^{2}}{r_{1}(\sigma)} d \sigma & \ldots & \int_{t_{0}}^{t} \frac{3}{2} \frac{\left(\sigma-t_{0}\right)^{2}}{r_{n_{L}}(\sigma)} d \sigma \\
\int_{t_{0}}^{t} \frac{1}{2} \frac{\left(\sigma-t_{0}\right)^{3}}{r_{1}(\sigma)} d \sigma & \ldots & \int_{t_{0}}^{t} \frac{1}{2} \frac{\left(\sigma-t_{0}\right)^{3}}{r_{n_{L}}(\sigma)} d \sigma
\end{array}\right]^{T},
$$

and $\phi_{C A}\left(t, t_{0}\right)$ and $\phi_{C C}\left(t, t_{0}\right)$ are omitted for the sake of simplicity, as they are not required in the paper.

\section{APPENDIX B}

\subsection{PROOF OF THEOREM 3}

Proof. Suppose that (11) is not observable on $\left[t_{0}, t_{f}\right]$. Then, it follows from Lemma 1 that the observability Gramian is not positive definite and therefore

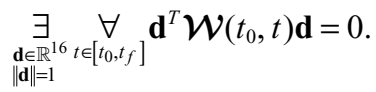

Expanding (49) gives $\int_{t_{0}}^{t}\left\|\mathbf{C}(\sigma) \phi\left(\sigma, t_{0}\right) \mathbf{d}\right\|^{2} d \sigma=0$ which, in particular, implies that

$$
\mathbf{C}(t) \phi\left(t, t_{0}\right) \mathbf{d}=\mathbf{0}
$$

for all $t \in\left[t_{0}, t_{f}\right]$. Let

$$
\mathbf{d}=\left[\begin{array}{llllll}
\mathbf{d}_{1}^{T} & \ldots & \mathbf{d}_{4}^{T} & d_{5} & \ldots & d_{8}
\end{array}\right]^{T} \in \mathbb{R}^{16},
$$

$\mathbf{d}_{1}, \ldots, \mathbf{d}_{4} \in \mathbb{R}^{3}, d_{5}, \ldots, d_{8} \in \mathbb{R}$. Notice that, for $t=t_{0},(50)$ reads as

$$
\left[\begin{array}{c}
\mathbf{d}_{4} \\
\mathbf{C}_{1}\left(t_{0}\right) \mathbf{d}_{1}+\mathbf{d}_{4}
\end{array}\right]=\mathbf{0} .
$$

Therefore, not only it must be $\mathbf{d}_{4}=\mathbf{0}$ but also $\mathbf{d}_{1}=\mathbf{0}$ or, if $\mathbf{d}_{1}$ is not null, it must be orthogonal to the plane defined by the landmarks, i.e., either $\mathbf{d}_{1}=\mathbf{0}$ or $\mathbf{d}_{1}$ is parallel to $\mathbf{d}^{\perp}$. From (50) it follows that

$$
\frac{d}{d t}\left[\mathbf{C}(t) \phi\left(t, t_{0}\right) \mathbf{d}\right]=\mathbf{0}
$$

for all $t \in\left[t_{0}, t_{f}\right]$, which, in particular, for $t=t_{0}$, implies that

$$
\left[\begin{array}{ll}
-\mathbf{s}_{1}^{T} & 1 \\
-\mathbf{s}_{2}^{T} & 1 \\
-\mathbf{s}_{3}^{T} & 1
\end{array}\right]\left[\begin{array}{l}
\mathbf{d}_{2} \\
d_{5}
\end{array}\right]=\mathbf{0} \text {. }
$$


Since the landmarks are noncolinear, the only nontrivial solution of (52) is such that $\mathbf{d}_{2}$ is parallel to $\mathbf{d}^{\perp}$. From (51) it follows that $\frac{d^{2}}{d t^{2}}\left[\mathbf{C}(t) \phi\left(t, t_{0}\right) \mathbf{d}\right]=\mathbf{0}$ for all $t \in\left[t_{0}, t_{f}\right]$, which, in particular, for $t=t_{0}$, implies that

$$
\left[\begin{array}{ll}
-\mathbf{s}_{1}^{T} & 1 \\
-\mathbf{s}_{2}^{T} & 1 \\
-\mathbf{s}_{3}^{T} & 1
\end{array}\right]\left[\begin{array}{l}
\mathbf{d}_{3} \\
d_{6}
\end{array}\right]=\mathbf{0} .
$$

Again, as the landmarks are noncolinear, the only nontrivial solution of (53) is such that $\mathbf{d}_{3}$ is parallel to $\mathbf{d}^{\perp}$. Now, expanding (51) allows us to write

$$
\begin{aligned}
& \frac{1}{r_{i}(t)} \mathbf{u}^{[1]}\left(t, t_{0}\right) \cdot \mathbf{d}_{1} \\
& \quad+\frac{1}{r_{i}(t)}\left[-\mathbf{s}_{i}+\left(t-t_{0}\right) \mathbf{u}^{[1]}\left(t, t_{0}\right)+\mathbf{u}^{[2]}\left(t, t_{0}\right)\right] \cdot \mathbf{d}_{2} \\
& \quad+\frac{t-t_{0}}{r_{i}(t)}\left[-\mathbf{s}_{i}+\frac{t-t_{0}}{2} \mathbf{u}^{[1]}\left(t, t_{0}\right)+\mathbf{u}^{[2]}\left(t, t_{0}\right)\right] \cdot \mathbf{d}_{3} \\
& \quad+\frac{1}{r_{i}(t)} d_{5}+\frac{1}{r_{i}(t)}\left(t-t_{0}\right) d_{6} \\
& \quad+\frac{1}{r_{i}(t)} \frac{3}{2}\left(t-t_{0}\right)^{2} d_{7}+\frac{1}{r_{i}(t)} \frac{1}{2}\left(t-t_{0}\right)^{3} d_{8}=0
\end{aligned}
$$

for all $t \in\left[t_{0}, t_{f}\right], i=1,2,3$, which in turn implies that

$$
\begin{gathered}
\mathbf{u}^{[1]}\left(t, t_{0}\right) \cdot \mathbf{d}_{1}+\left[-\mathbf{s}_{i}+\left(t-t_{0}\right) \mathbf{u}^{[1]}\left(t, t_{0}\right)+\mathbf{u}^{[2]}\left(t, t_{0}\right)\right] \cdot \mathbf{d}_{2} \\
+\left(t-t_{0}\right)\left[-\mathbf{s}_{i}+\frac{t-t_{0}}{2} \mathbf{u}^{[1]}\left(t, t_{0}\right)+\mathbf{u}^{[2]}\left(t, t_{0}\right)\right] \cdot \mathbf{d}_{3} \\
+d_{5}+\left(t-t_{0}\right) d_{6}+\frac{3}{2}\left(t-t_{0}\right)^{2} d_{7}+\frac{1}{2}\left(t-t_{0}\right)^{3} d_{8}=0
\end{gathered}
$$

for all $t \in\left[t_{0}, t_{f}\right], i=1,2,3$. Now, integrating both sides of (54) gives

$$
\begin{aligned}
& \mathbf{u}^{[2]}\left(t, t_{0}\right) \cdot \mathbf{d}_{1}+\left[-\left(t-t_{0}\right) \mathbf{s}_{i}+\left(t-t_{0}\right) \mathbf{u}^{[2]}\left(t, t_{0}\right)\right] \cdot \mathbf{d}_{2} \\
& +\left[-\frac{\left(t-t_{0}\right)^{2}}{2} \mathbf{s}_{i}+\frac{\left(t-t_{0}\right)^{2}}{2} \mathbf{u}^{[2]}\left(t, t_{0}\right)\right] \cdot \mathbf{d}_{3} \\
& +\left(t-t_{0}\right) d_{5}+\frac{\left(t-t_{0}\right)^{2}}{2} d_{6}+\frac{1}{2}\left(t-t_{0}\right)^{3} d_{7} \\
& +\frac{1}{8}\left(t-t_{0}\right)^{4} d_{8}=0
\end{aligned}
$$

for all $t \in\left[t_{0}, t_{f}\right], i=1,2,3$, or, equivalently,

$$
\begin{aligned}
& \mathbf{u}^{[2]}\left(t, t_{0}\right) \cdot \mathbf{d}_{1}+\left[\left(t-t_{0}\right) \mathbf{u}^{[2]}\left(t, t_{0}\right)\right] \cdot \mathbf{d}_{2} \\
& \quad+\left[\frac{\left(t-t_{0}\right)^{2}}{2} \mathbf{u}^{[2]}\left(t, t_{0}\right)\right] \cdot \mathbf{d}_{3}+\left(t-t_{0}\right)\left(d_{5}-\mathbf{s}_{i} \cdot \mathbf{d}_{2}\right) \\
& \quad+\frac{\left(t-t_{0}\right)^{2}}{2}\left(d_{6}-\mathbf{s}_{i} \cdot \mathbf{d}_{3}\right)+\frac{1}{2}\left(t-t_{0}\right)^{3} d_{7}+\frac{1}{8}\left(t-t_{0}\right)^{4} d_{8}=0 .
\end{aligned}
$$

From (52) and (53) it is possible to simplify (55) as

$$
\begin{aligned}
\mathbf{u}^{[2]}\left(t, t_{0}\right) \cdot \mathbf{d}_{1}+\left[\left(t-t_{0}\right) \mathbf{u}^{[2]}\left(t, t_{0}\right)\right] \cdot \mathbf{d}_{2} \\
+\left[\frac{\left(t-t_{0}\right)^{2}}{2} \mathbf{u}^{[2]}\left(t, t_{0}\right)\right] \cdot \mathbf{d}_{3} \\
+\frac{1}{2}\left(t-t_{0}\right)^{3} d_{7}+\frac{1}{8}\left(t-t_{0}\right)^{4} d_{8}=0,
\end{aligned}
$$

for all $t \in\left[t_{0}, t_{f}\right]$, where $\mathbf{d}_{1}, \mathbf{d}_{2}$, and $\mathbf{d}_{3}$ are either zero or parallel to $\mathbf{d}^{\perp}$. However, $\mathbf{d}_{1}=\mathbf{d}_{2}=\mathbf{d}_{3}=\mathbf{0}$ is not a solution. Indeed, if that was the case, from (52) and (53) it would follow that $d_{5}=d_{6}=0$ and, it would be impossible to satisfy (56) with a non-null $\mathbf{d}$, as $\left(t-t_{0}\right)^{3}$ and $\left(t-t_{0}\right)^{4}$ are linearly independent. Then, at least one of the sets of functions

$$
\begin{aligned}
& \left\{\left(t-t_{0}\right)^{3},\left(t-t_{0}\right)^{4},\left[\mathbf{u}^{[2]}\left(t, t_{0}\right)\right] \cdot \mathbf{d}^{\perp}\right\}, \\
& \left\{\left(t-t_{0}\right)^{3},\left(t-t_{0}\right)^{4},\left(t-t_{0}\right)\left[\mathbf{u}^{[2]}\left(t, t_{0}\right)\right] \cdot \mathbf{d}^{\perp}\right\},
\end{aligned}
$$

or

$$
\left\{\left(t-t_{0}\right)^{3},\left(t-t_{0}\right)^{4},\left(t-t_{0}\right)^{2}\left[\mathbf{u}^{[2]}\left(t, t_{0}\right)\right] \cdot \mathbf{d}^{\perp}\right\}
$$

is not linearly independent on $\left[t_{0}, t_{f}\right]$, from which follows that set of functions $\mathcal{F}_{3}$ is not linearly independent. Then, if the set of functions $\mathcal{F}_{3}$ is linearly independent on $t \in\left[t_{0}, t_{f}\right]$, (11) is observable on $\left[t_{0}, t_{f}\right]$.

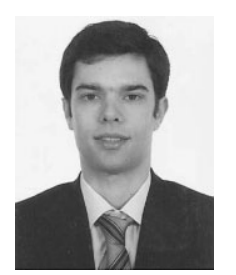

Pedro Batista received, in 2005, the Licenciatura degree in Electrical and Computer Engineering from Instituto Superior Técnico (IST), Lisbon, Portugal, and the Ph.D. degree from the same university in 2010. From 2004 to 2006 he was Monitor with the Department of Mathematics of IST and he is currently Invited Assistant Professor with the Department of Electrical and Computer Engineering of IST. He has received the Diploma de Mérito twice during his graduation and his Ph.D. thesis was awarded the best Robotics Ph.D. thesis award by the Portuguese Society of Robotics. His research interests include sensor-based navigation and control of autonomous vehicles

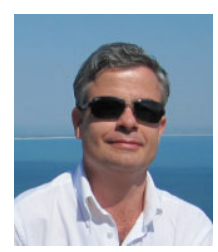

Carlos Silvestre received the Licenciatura degree in Electrical Engineering from the Instituto Superior Tecnico (IST) of Lisbon, Portugal, in 1987 and the M.Sc. degree in Electrical Engineering and the Ph.D. degree in Control Science from the same school in 1991 and 2000, respectively. In 2011 he received the Habilitation in Electrical Engineering and Computers also from IST. Since 2000, he is with the Department of Electrical Engineering of the Instituto Superior Tecnico, where he is 
currently Associate Professor of Control and Robotics in leave. Since 2012 he is Associate Professor of the Department o Electrical and Computers Engineering of the Faculty of Science and Technology of the University of Macau. Over the past years, he has conducted research on the subjects of navigation guidance and control of air and underwater robots. His research interests include linear and nonlinear control theory, coordinated control of multiple vehicles, gain scheduled control, integrated design of guidance and control systems, inertial navigation systems, and mission control and real time architectures for complex autonomous systems with applications to unmanned air and underwater vehicles.

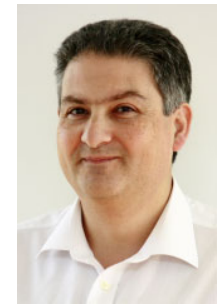

Paulo Oliveira completed the Ph.D. in 2002 from the Instituto Superior Técnico, Lisbon, Portugal. He is Associate Professor of the Department of Mechanical Engineering and of the Instituto Superior Técnico, Lisbon, Portugal and researcher in the Institute for Systems and RoboticsAssociated Laboratory, Lisbon, Portugal. The areas of scientific activity are Robotics and Autonomous Vehicles with special focus on the fields of Sensor Fusion, Navigation, Positioning, and Signal Processing. He participated in more than 15 Portuguese and European Research projects, in the last 20 years. 